\title{
UNIFORM BOUNDS FOR THE NUMBER OF RATIONAL POINTS ON CURVES OF SMALL MORDELL-WEIL RANK
}

\author{
ERIC KATZ, JOSEPH RABINOFF, AND DAVID ZUREICK-BROWN \\ Dedicated to the memory of Robert Coleman.
}

\begin{abstract}
Let $X$ be a curve of genus $g \geq 2$ over a number field $F$ of degree $d=[F: \mathbf{Q}]$. The conjectural existence of a uniform bound $N(g, d)$ on the number $\# X(F)$ of $F$-rational points of $X$ is an outstanding open problem in arithmetic geometry, known by Caporaso, Harris, and Mazur to follow from the Bombieri-Lang conjecture. A related conjecture posits the existence of a uniform bound $N_{\text {tors, } \uparrow}(g, d)$ on the number of geometric torsion points of the Jacobian $J$ of $X$ which lie on the image of $X$ under an Abel-Jacobi map. For fixed $X$ this quantity was conjectured to be finite by the Manin-Mumford conjecture, and was proved to be so by Raynaud.

We give an explicit uniform bound on $\# X(F)$ when $X$ has Mordell-Weil rank $r \leq g-3$. This generalizes recent work of Stoll on uniform bounds for hyperelliptic curves of small rank to arbitrary curves. Using the same techniques, we give an explicit, unconditional uniform bound on the number of $F$-rational torsion points of $J$ lying on the image of $X$ under an Abel-Jacobi map. We also give an explicit uniform bound on the number of geometric torsion points of $J$ lying on $X$ when the reduction type of $X$ is highly degenerate.

Our methods combine Chabauty-Coleman's $p$-adic integration, non-Archimedean potential theory on Berkovich curves, and the theory of linear systems and divisors on metric graphs.
\end{abstract}

\section{INTRODUCTION}

Let $X$ be a curve of genus $g \geq 2$ over a number field $F$ of degree $d=[F: \mathrm{Q}]$. The conjectural existence of a uniform bound $N(g, d)$ on the number $\# X(F)$ of $F$-rational points of $X$ is an outstanding open problem in arithmetic geometry, known by [CHM97] to follow from the Bombieri-Lang conjecture. A related conjecture posits the existence of a uniform bound $N_{\text {tors } \uparrow \uparrow}(g, d)$ on the number of geometric torsion points of the Jacobian of $X$ which lie on the image of $X$ under an Abel-Jacobi map. For fixed $X$ this quantity was conjectured to be finite by the Manin-Mumford conjecture, and was proved to be so by Raynaud Ray83.

In this paper we obtain both kinds of uniform bounds for large classes of curves where uniformity was previously unknown. To do so, we combine Chabauty and Coleman's method of $p$-adic integration, potential theory on Berkovich curves, and the theory of linear systems and divisors on metric graphs. The main theorems are as follows.

Theorem 1.1. Let $d \geq 1$ and $g \geq 3$ be integers. There exists an explicit constant $N(g, d)$ such that for any number field $F$ of degree $d$ and any smooth, proper, geometrically connected 
genus $g$ curve $X / F$ of Mordell-Weil rank at most $g-3$, we have

$$
\# X(F) \leq N(g, d)
$$

The Mordell-Weil rank is by definition the rank of the finitely generated abelian group $J(F)$, where $J$ is the Jacobian of $X$. Theorem 1.1 is an improvement on a theorem of Stoll [Sto13], which applies to hyperelliptic $X$. The methods used to prove Theorem 1.1 are largely inspired by Stoll's ideas. (See Section 1.1 for a discussion of Stoll's results and their relation to this paper.)

There are any number of different ways of expressing the bound $N(g, d)$. For instance, in the case $F=\mathbf{Q}$, we can take

$$
N(g, 1)=84 g^{2}-98 g+28
$$

by applying Theorem 5.1 with $K=\mathrm{Q}_{3}$ and by using (4.6).

Next, we define an equivalence relation on the set of $\bar{F}$-points of a curve $X / F$ as follows: we say that two points $P, Q$ are equivalent if $m P$ is linearly equivalent to $m Q$ on $X_{\bar{F}}$ for some integer $m \geq 1$. We define a torsion packet to be an equivalence class under this relation. Equivalently, a torsion packet is the inverse image of the group of geometric torsion points of the Jacobian $J$ of $X$ under an Abel-Jacobi map $X_{\bar{F}} \hookrightarrow J_{\bar{F}}$. Replacing $\bar{F}$ with $F$, one has a notion of a rational torsion packet as well. As mentioned above, Raynaud Ray83 proved that every torsion packet of a curve is finite. Many additional proofs, with an assortment of techniques and generalizations, were given later by [Bui96, Col87, Hin88, Ul198, [PZ08], and others. Several of these proofs rely on $p$-adic methods, with the method of Coleman being particularly closely related to ours.

A uniform bound on the size of the torsion packets of a curve of genus $g \geq 2$ is expected but still conjectural. We offer two results in this direction. The first unconditional result concerns rational torsion packets and is proved along with Theorem 1.1. To our knowledge, no uniformity result was previously known even in this case for general curves (for hyperelliptic curves it follows from [Sto13, Theorem 8.1]).

Theorem 1.2. Let $d \geq 1$ and $g \geq 3$ be integers. There exists an explicit constant $N_{\text {tors }}(g, d)$ such that for any number field $F$ of degree $d$, any smooth, proper, geometrically connected genus $g$ curve $X / F$, and any Abel-Jacobi embedding $\iota: X \hookrightarrow J$ into its Jacobian (defined over $F$ ), we have

$$
\# \iota^{-1}\left(J(F)_{\text {tors }}\right) \leq N_{\text {tors }}(g, d) .
$$

In fact, one may take $N_{\text {tors }}(g, d)=N(g, d)$, the same constant in Theorem 1.1. Note that here there is no restriction on the Mordell-Weil rank.

The second result concerns (geometric) torsion packets. It involves the following restriction on the reduction type. Let $F$ be a number field, and let $\mathfrak{p}$ be a finite prime of $F$. Let $X$ be a smooth, proper, geometrically connected curve of genus $g \geq 2$ over $F$. Let $\mathfrak{X}$ be the stable model of $X$ over an algebraic closure of $F_{\mathfrak{p}}$. For each irreducible component $C$ of the special fiber $\mathfrak{X}_{s}$ of $\mathfrak{X}$, let $g(C)$ denote its geometric genus, and let $n_{C}$ denote the number points of 
the normalization of $C$ mapping to nodal points of $\mathfrak{X}_{s}$. We say that $X$ satisfies condition $(\dagger)$ at $\mathfrak{p}$ provided that

$$
g>2 g(C)+n_{C}
$$

for each component $C$ of $\mathfrak{X}_{s}$.

Theorem 1.3. Let $d \geq 1$ and $g \geq 4$ be integers. There exists an explicit constant $N_{\text {tors }, \dagger}(g, d)$ such that for any number field $F$ of degree $d$ and any smooth, proper, geometrically connected genus $g$ curve $X / F$ which satisfies condition $(\dagger)$ at some prime $\mathfrak{p}$ of $F$, we have

$$
\# \iota^{-1}\left(J(\bar{F})_{\text {tors }}\right) \leq N_{\text {tors }, \dagger}(g, d) .
$$

for any Abel-Jacobi embedding $\iota: X_{\bar{F}} \hookrightarrow J_{\bar{F}}$ of $X_{\bar{F}}$ into its Jacobian.

The condition $(\dagger)$ is satisfied at $\mathfrak{p}$, for instance, when $X$ has totally degenerate trivalent stable reduction over $F_{\mathfrak{p}}$. One can take

$$
N_{\text {tors }, \dagger}(g, d)=\left(16 g^{2}-12 g\right) N_{2}\left(\left(4 d \cdot 7^{2 g^{2}+g+1}\right)^{-1}, 2 g-2\right),
$$

where

$$
N_{2}\left(s, N_{0}\right)=\min \left\{N \in \mathbf{Z}_{\geq 1}: s\left(n-N_{0}\right)>\left\lfloor\log _{2}(n)\right\rfloor \forall n \geq N\right\} .
$$

See Theorem 5.5 for a more precise statement.

A uniform bound as in Theorem 1.3 for the size of geometric torsion packets was previously known (see [Bui96]) for curves of good reduction at a fixed prime $\mathfrak{p}$. This result uses work of Coleman [Col87], who also deduces uniform bounds in many situations, still in the good reduction case: for instance, if $X / \mathrm{Q}$ has ordinary good reduction at $p$ and its Jacobian $J$ has potential complex multiplication, then $\# \iota^{-1}\left(J(\overline{\mathbf{Q}})_{\text {tors }}\right) \leq g p$. Theorem 1.3, on the other hand, applies to curves with highly degenerate reduction, and hence approaches the uniform Manin-Mumford conjecture from the other extreme. It is also independent of the residue characteristic of $F_{\mathfrak{p}}$.

The full power of the general machinery developed in this paper is needed for the proof of Theorem 1.3, which is striking in that it uses $p$-adic integration techniques to bound the number of geometric torsion points. Whereas Theorems 1.1 and 1.2 only involve integration on discs and annuli, which was Stoll's idea, Theorem 1.3 requires integrating over finitely many wide open subdomains which cover $X^{\text {an }}$, and, as such, is more subtle. (See Section 1.1 below for a more detailed summary of the proofs.)

Remark 1.5. One expects that the Mordell-Weil rank of the Jacobian of a curve is usually 0 or 1 . In practice one needs a family of curves over a rational base to even make this precise. One therefore often restricts to families of hyperelliptic (or sometimes low genus plane) curves, in which case there are very recent partial results: see [BS13] (elliptic curves), [BG13] (Jacobians of hyperelliptic curves), and [Tho15] (certain plane quartics). Combining these rank results with Chabauty's method and other techniques, several recent results prove that the uniformity conjecture holds for a random curve (in that there are no "nonobvious" points): see [PS14, Bha13, SW13, BGW13] (see [Ho14] for a recent survey). 
1.1. Overview of the proofs. Our central technique is Chabauty and Coleman's method of $p$-adic integration. In a 1941 paper, Chabauty [Cha41] proved the Mordell conjecture in the special case of curves with Mordell-Weil rank at most $g-1$, via a study of the $p$-adic Lie theory of the Jacobian of $X$. Four decades later, Coleman Col85 made Chabauty's method explicit: he proved that for a curve $X / \mathbf{Q}$ of genus $g \geq 2$, rank $r<g$, and a prime $p>2 g$ of good reduction,

$$
\# X(\mathbf{Q}) \leq \# X\left(\mathbf{F}_{p}\right)+2 g-2 .
$$

Coleman's method has been refined by many authors: these authors [LT02, MP12, Sto06, KZB13, allow $X$ to have bad reduction at $p$ and improve the $2 g-2$ to $2 r$, [Sik09, Par14] generalize to symmetric powers of curves, and a large body of work by many authors allow one to explicitly execute this method in Magma for any particular curve of low genus and low rank, frequently allowing one to compute $X(\mathbf{Q})$ exactly.

Our starting point for proving Theorems 1.1] and 1.2] is the recent progress of Stoll [Sto13], who proves that for any hyperelliptic curve $X / \mathrm{Q}$ with Jacobian of rank $r \leq g-3$,

$$
\# X(\mathbf{Q}) \leq 8(r+4)(g-1)+\max \{1,4 r\} \cdot g .
$$

While this bound still depends on $r$ and $g$, its independence from $p$ is a substantial improvement. This improvement is made possible by fixing a prime $p$ (generally small and odd) and considering curves $X / \mathbf{Q}_{p}$ with arbitrary reduction type. Stoll's bold idea is to decompose $X\left(\mathbf{Q}_{p}\right)$ into a disjoint union of residue discs and residue annuli and to execute Chabauty's method on both. The decomposition is achieved by performing a careful analysis of the minimal regular model of $X$ over $\mathbf{Z}_{p}$. Bounding zeros of integrals on annuli is somewhat subtle: monodromy becomes an issue, and a key technical feature of Stoll's work is his analysis and comparison of analytic continuation and the emergent $p$-adic logarithms. Stoll's method exploits the description of differentials on a hyperelliptic curve as $f(x) d x / y$; using an explicit calculation, he is able to analyze the zeroes of the resulting integral directly via Newton polygons.

In contrast, a differential on a typical curve may lack an explicit description, and a direct, explicit analysis is impervious to classical methods. Moreover, one cannot hope to attain any kind of geometric bound as in Theorem 1.3 by analyzing $p$-adic integrals on discs and annuli alone, as the antiderivative of an analytic function on a disc or annulus may well have infinitely many geometric zeros. This is where potential theory on Berkovich analytic curves and the theory of linear systems on metric graphs becomes useful. To be clear, the inputs into the proofs of Theorems 1.1 and 1.2 are Stoll's bounds [Sto13, Proposition 5.3] on the number of discs and annuli covering $X\left(\mathbf{Q}_{p}\right)$, and a new method of bounding the zeros of an integral on an open annulus (Corollary 4.18). As mentioned before, the full power of the general machinery developed in this paper is needed for the proof of Theorem 1.3. only a fraction of it (namely, Section 2 along with Lemma 4.15) is needed for Corollary 4.18.

Let us give an overview of our methods. They are entirely geometric, so we work over the field $\mathbf{C}_{p}$, the completion of an algebraic closure of $\mathbf{Q}_{p}$. Let $X$ be a curve over $\mathbf{C}_{p}$ of genus $g \geq 2$, and let $X^{\text {an }}$ denote the analytification of $X$, in the sense of Berkovich [Ber90]. This 
is a reasonable topological space in that it deformation retracts onto a finite metric graph $\Gamma \subset X^{\text {an }}$ called a skeleton, whose combinatorics is controlled by a semistable model of $X$. (As $\mathbf{C}_{p}$ is algebraically closed, such a model exists.) If $f$ is a nonzero rational function on $X$, then $-\log |f|$ is a piecewise affine function on $\Gamma$ with integer slopes. Letting $\tau: X^{\text {an }} \rightarrow \Gamma$ denote the deformation retraction, the inverse image $\tau^{-1}(V)$ of a small neighborhood $V$ of a vertex $v$ of $\Gamma$ is a basic wide open subdomain in the sense of Coleman [Col89, Section 3]. One can cover $X^{\text {an }}$ by finitely many basic wide open subdomains.

Our proof (roughly) proceeds by using the following steps.

(1) Let $f$ be a nonzero analytic function on a basic wide open $U$ with central vertex $v$. A basic fact from potential theory on $X^{\text {an }}$ implies that $\operatorname{deg}(\operatorname{div}(f))$ can be calculated by summing the slopes of $-\log |f|$ along the incoming edges at $v$ (see Proposition 2.22).

(2) Let $\omega$ be an exact differential form on $U$, and let $f=\int \omega$ be an antiderivative. A Newton polygon calculation (Proposition 4.7) relates the slopes of $-\log |f|$ with the slopes of $-\log \|\omega\|$. Here $\|\omega\|$ is the norm of $\omega$ with respect to the canonical metric on $\Omega_{X / \mathbf{C}_{p}}^{1}$, described in Section 2.4. The "error term" $N_{p}(\cdot, \cdot)$ appearing in (1.4) is introduced at this point.

(3) Suppose now that $\omega$ is a global differential form on $X$. Then the restriction $F$ of $-\log \|\omega\|$ to $\Gamma$ is a "section of the tropical canonical sheaf," in that $\operatorname{div}(F)+K_{\Gamma} \geq 0$, where $K_{\Gamma}$ is the canonical divisor on the graph $\Gamma$. This is a consequence of the slope formula (otherwise known as the Poincaré-Lelong formula) for line bundles on Berkovich curves, which we prove in Theorem 2.6.

(4) With $\omega$ and $F$ as above, we use a combinatorial argument (Lemma 4.15) about linear systems on vertex-weighted metric graphs to bound the slopes of $F$ in terms of the genus of the graph $\Gamma$, which is bounded by the genus of the curve. This step plays the role of the usual Riemann-Roch part of the Chabauty-Coleman argument. It also plays the role of [Sto13, Corollary 6.7], which is proved using explicit calculations on hyperelliptic curves.

(5) Using Coleman's calculation of the de Rham cohomology of a wide open subdomain $U$, under the restriction $(\dagger)$ we can produce a nonzero global differential form $\omega$ which is exact on $U$. Combining the above steps then provides a uniform bound on the number of zeros of $\int \omega$ on $U$. Covering $X^{\text {an }}$ by such wide opens $U$ yields Theorem 1.3, as the integral of any differential form vanishes on torsion points.

(6) An open annulus is a simple kind of wide open subdomain. Specializing the above results to annuli (Corollary 4.18) gives the generalization of [Sto13, Proposition 7.7] needed to prove Theorems 1.1 and 1.2 by using [Sto13, Proposition 5.3].

It should be mentioned that in principle one can avoid the Berkovich language by using intersection theory on semistable curves, but this leads to fussy arguments and frequent base changes and at certain points is very difficult to do. We hope the reader will agree that the analytic framework is much more natural.

In the summary above we have suppressed a major technical difficulty. By an "antiderivative" of $\omega$, we always mean an analytic function $f$ such that $d f=\omega$. The definite integral 
$\int_{x}^{y} f$ is then defined to be $f(y)-f(x)$; this is what is needed for Newton polygons and potential theory. However, for curves of bad reduction this does not generally coincide with the abelian integration used in the Chabauty-Coleman method, defined in terms of a $p$-adic logarithm on the Jacobian. Indeed, the former kind of integration will have $p$-adic periods, whereas the latter cannot. This was realized by Stoll [Sto13, who found a way to compare the integrals on annuli. A systematic comparison between these integration theories in general, given in Section 3, should be of independent interest.

1.2. Organization of paper. In Section 2, we recall several basic facts about Berkovich curves, and we develop the $p$-adic analytic machinery that we will need. The main features are the following: Theorem 2.6, a generalization of the slope formula [BPR13, Theorem 5.15] to sections of formally metrized line bundles; a careful treatment of Rosenlicht differentials, a generalization of the relative dualizing sheaf to a semistable curve over a possibly nondiscretely valued field, needed in order to define the norm $\|\omega\|$ of a differential; and Coleman's calculation (Theorem 2.24) of the de Rham cohomology of a basic wide open subdomain.

In Section 3, we recall the basic properties of the Berkovich-Coleman integral and the abelian integral in our somewhat restricted setting. We then prove a result (Proposition 3.28) comparing the two: essentially, the difference is controlled by the tropical Abel-Jacobi map. The non-Archimedean uniformization theory of abelian varieties plays a central role here.

In Section 4, we explicitly bound the slopes of an analytic function $f$ on an annulus in terms of the slopes of $\omega=d f$ (Proposition 4.7) and deduce, via a quick combinatorial argument (Lemma 4.14), a bound on the number of zeroes of the integral of an exact differential on a wide open (Theorem 4.17).

Finally, in Section 5, we put everything together, proving our main theorems on uniform bounds.

\section{Berkovich CURVES}

In this section we develop the basic geometric facts about analytic curves over nonArchimedean fields that will be used below.

2.1. General notation. We will use the following notations for non-Archimedean fields, in this section only. In subsequent sections we will generally restrict our attention to $\mathbf{C}_{p}$.

$K \quad$ A field that is complete with respect to a nontrivial, non-Archimedean valuation.

val $: K^{\times} \rightarrow \mathbf{R} \cup\{\infty\}$, the fixed valuation on $K$.

$|\cdot|=\exp (-\operatorname{val}(\cdot))$, a corresponding absolute value.

$R=\mathscr{O}_{K}$, the valuation ring of $K$.

$k \quad$ The residue field of $K$.

$\Lambda=\operatorname{val}\left(K^{\times}\right) \subset \mathbf{R}$, the value group of $K$.

$\sqrt{\Lambda}$ The saturation of $\Lambda$.

Let $X$ be a proper $K$-scheme, and let $\mathfrak{X}$ be a proper, flat $R$-model of $X$. We use the following notations: 
$X^{\text {an }} \quad$ The analytification of $X$, in the sense of Berkovich [Ber90].

$\mathscr{H}(x)$ The completed residue field at a point $x \in X^{\text {an }}$.

$\mathfrak{X}_{k} \quad$ The special fiber of $\mathfrak{X}$.

red $\quad: X^{\text {an }} \rightarrow \mathfrak{X}_{k}$, the reduction or specialization map.

The completed residue field is a valued field extension of $K$. For $x \in X^{\text {an }}$ the reduction $\operatorname{red}(x)$ is defined by applying the valuative criterion of properness to the canonical $K$-morphism $\operatorname{Spec}(\mathscr{H}(x)) \rightarrow \mathfrak{X}$. The reduction map is anticontinuous, in that the inverse image of a closed set is open.

2.2. Skeletons. Here we fix our notions regarding non-Archimedean analytic curves and their skeletons. We adhere closely to the treatment in BPR13, our primary reference.

Let $X$ be a smooth, proper, geometrically connected $K$-curve. We say that a semistable $R$ model $\mathfrak{X}$ is split if the $G_{k}$-action on the dual graph of $\mathfrak{X}_{k^{\text {sep }}}$ is trivial, where $G_{k}=\operatorname{Gal}\left(k^{\text {sep }} / k\right)$. Equivalently, we require that each component of $\mathfrak{X}_{k^{\text {sep }}}$ be defined over $k$, that all nodes of $\mathfrak{X}_{k^{\text {sep }}}$ be $k$-rational, and that the completed local ring of $\mathfrak{X}_{k^{\text {sep }}}$ at a node be isomorphic to $k \llbracket R, S \rrbracket /(R S)$. (The final condition rules out the possibility that $G_{k}$ acts via an involution on a loop edge, i.e., that it interchanges "tangent directions" at the node.) Let $\mathfrak{X}$ be a split semistable $R$-model of $X$. We will use the following notations for the structure theory of $X^{\text {an }}$ :

$\Gamma_{\mathfrak{X}} \subset X^{\text {an }}$, the skeleton associated to $\mathfrak{X}$.

$\tau: X^{\text {an }} \rightarrow \Gamma_{\mathfrak{X}}$, the retraction to the skeleton.

$g(x)$ The genus of a type-2 point $x \in X^{\text {an }}$.

In general, a skeleton of $X$ is a skeleton $\Gamma=\Gamma_{\mathfrak{X}}$ corresponding to some split semistable model $\mathfrak{X}$ of $X$. It is a $\Lambda$-metric graph (a metric graph with edge lengths in $\Lambda$ ) whose vertices correspond bijectively to the generic points of $\mathfrak{X}_{k}$ in the following way: if $x \in \Gamma_{\mathfrak{X}}$ is a vertex, then $\zeta=\operatorname{red}(x)$ is a generic point of $\mathfrak{X}_{k}$, and $\operatorname{red}^{-1}(\zeta)=\{x\}$. The edges of $\Gamma_{\mathfrak{X}}$ correspond to the singular points of $\mathfrak{X}_{k}$, as follows. For $\varpi \in R$ nonzero we let

$$
\mathbf{S}(\varpi)_{+}=\left\{\xi \in \mathbf{G}_{m}^{\text {an }}:|\varpi|<|T(\xi)|<1\right\},
$$

the open annulus of modulus $|\varpi|$. Here $T$ is a parameter on $\mathbf{G}_{m}$; that is, $\mathbf{G}_{m}=\operatorname{Spec}\left(K\left[T, T^{-1}\right]\right)$. If $\widetilde{x} \in \mathfrak{X}_{k}$ is a node, then $\operatorname{red}^{-1}(\widetilde{x}) \cong \mathbf{S}(\varpi)_{+}$for some $\varpi \in R$ with $|\varpi| \in(0,1)$; the open edge $e$ of $\Gamma_{\mathfrak{X}}$ corresponding to $\widetilde{x}$ is the skeleton of the annulus $\mathbf{S}(\varpi)_{+}$(see Section 4.1), and the length of $e$ is the logarithmic modulus $\operatorname{val}(\varpi) \in \Lambda$ of $\mathbf{S}(\varpi)_{+}$, which is an isomorphism invariant.

The weight $g(x)$ of a vertex $x \in \Gamma_{\mathfrak{X}}$ is defined to be the genus of the type- 2 point $x \in X^{\text {an }}$, which in turn is the geometric genus of the corresponding component of $\mathfrak{X}_{k}$. We have the basic identity

$$
g(X)=h_{1}\left(\Gamma_{\mathfrak{X}}\right)+\sum_{x \in \Gamma_{\mathfrak{X}}} g(x),
$$

where $g(X)$ is the genus of the curve $X$, and $h_{1}\left(\Gamma_{\mathfrak{X}}\right)=\operatorname{dim}_{\mathbf{Q}} H_{1}\left(\Gamma_{\mathfrak{X}}, \mathbf{Q}\right)$ is the first (singular) Betti number of $\Gamma_{\mathfrak{X}}$. 
Any curve admits a split semistable model (and hence a skeleton) after potentially making a finite extension of the ground field $K$, of degree bounded by the genus (see the proof of Theorem 5.5). If $X$ has a skeleton, then it has a minimal skeleton, which comes from a stable $R$-model $\mathfrak{X}$ of $X$. If $g \geq 2$, then the minimal skeleton is unique, and we denote it by $\Gamma_{\min }$. The vertices of $\Gamma_{\min }$ are the points of $X^{\text {an }}$ of nonzero genus and the points of $\Gamma_{\min }$ of valency greater than 2 .

Remark 2.2. Let $X$ be a $K$-curve as above, let $\mathbf{C}_{K}=\widehat{\bar{K}}$ be the completion of the algebraic closure of $K$, and let $X^{\prime}=X_{\mathbf{C}_{K}}$ be the base change. If $\mathfrak{X}$ is a semistable model of $X$, then the base change $\mathfrak{X}^{\prime}$ to the ring of integers in $\mathbf{C}_{K}$ is a semistable model, which is necessarily split as the residue field of $\mathbf{C}_{K}$ is algebraically closed. The original model $\mathfrak{X}$ is split if and only if the natural action of $G_{K}=\operatorname{Gal}\left(K^{\mathrm{sep}} / K\right)$ on $X^{\text {an }}$ fixes $\Gamma_{\mathfrak{X}^{\prime}} \subset X^{\text {an }}$ pointwise, that is, if the skeleton $\Gamma_{\mathfrak{X}}$ is "defined over $K$." Indeed, Berkovich [Ber90] defines the skeleton associated to a nonsplit semistable model as the quotient of $\Gamma_{\mathfrak{X}}$ by the action of $G_{K}$. The split condition is necessary for the formal fibers over nodes in $\mathfrak{X}_{k}$ to be $K$-isomorphic to open annuli, which we use repeatedly.

Remark 2.3. Suppose that the genus $g$ of $X$ is at least 2 , let $\Gamma=\Gamma_{\min }$ be the minimal skeleton, and let $G$ be the underlying vertex-weighted (nonmetric) graph. Then $G$ is a connected graph of genus $g$ with the property that any vertex of valency 1 or 2 has positive weight. It is easy to see that there are finitely many isomorphism classes of such graphs. In other words, for fixed $g$, there are finitely many combinatorial types of minimal skeletons of curves of genus $g$. This crucial observation allows us to derive uniform bounds from stable models (see Section 4.2 for much more precise statements).

2.3. Metrized line bundles and the slope formula. In this section we assume that our non-Archimedean field $K$ is algebraically closed, which implies that $k$ is algebraically closed and $\Lambda=\sqrt{\Lambda}$. Let $X$ be a curve as in Section 2.2, and let $\Gamma \subset X^{\text {an }}$ be a skeleton which is not a point. There is a well-developed theory of divisors and linear equivalence on graphs and metric graphs, which we briefly recall here (see [Bak08] and the references therein for details). A tropical meromorphic function on $\Gamma$ is a continuous, piecewise affinelinear function $F: \Gamma \rightarrow \mathbf{R}$ with integral slopes. A divisor on $\Gamma$ is a formal sum of points of $\Gamma$; the group of divisors is denoted $\operatorname{Div}(\Gamma)$. The divisor of a meromorphic function $F$ is $\operatorname{div}(F)=\sum_{x \in \Gamma} \operatorname{ord}_{x}(F)(x)$, where $\operatorname{ord}_{x}(F)=-\sum_{v \in T_{x}(\Gamma)} d_{v} F(x), T_{x}(\Gamma)$ is the set of tangent directions at $x$, and $d_{v} F(x)$ is the slope of $F$ in the direction $v$. In other words, $\operatorname{ord}_{x}(F)$ is the sum of the incoming slopes of $F$ at $x$.

To reduce questions about curves to questions about skeletons, we will need to relate divisors on $X$ to divisors on $\Gamma$. The retraction map $\tau: X^{\text {an }} \rightarrow \Gamma$ extends by linearity to a map on divisors

$$
\tau_{*}: \operatorname{Div}(X) \longrightarrow \operatorname{Div}(\Gamma) .
$$

Theorem 2.4. Let $f$ be a nonzero meromorphic function on $X$, and let $F=-\left.\log |f|\right|_{\Gamma}$. Then $F$ is a tropical meromorphic function on $\Gamma$ and

$$
\operatorname{div}(F)=\tau_{*} \operatorname{div}(f) .
$$


Proof. This is a consequence of the slope formula for non-Archimedean curves (see [BPR13, Theorem 5.15]).

We will need a generalization of Theorem 2.4 that applies to a meromorphic section of a formally metrized line bundle. Theorem 2.6 below can in principle be extracted from Thuillier's Poincaré-Lelong formula [Thu05, Proposition 4.2.3], and indeed should be seen as a reformulation of [Thu05, Proposition 4.2.3], but it is easier to derive it from the slope formula as it appears in [BPR13, Theorem 5.15]. In the discretely valued case, a version of Theorem 2.6 can be found in Christensen's thesis [Chr13, Satz 1.3], with a similar proof.

The formal metric on a line bundle with an integral model is a basic construction in Arakelov theory, which we briefly recall. Let $\mathfrak{X}$ be an admissible formal $R$-scheme in the sense of [BL93], that is, a flat formal $R$-scheme of topological finite presentation. Let $X=\mathfrak{X}_{\eta}$ be the analytic generic fiber, a $K$-analytic space. Let $\mathfrak{L}$ be a line bundle on $\mathfrak{X}$, and let $L=\mathfrak{L}_{\eta}$, a line bundle on $X$. Let $s$ be a nonzero meromorphic section of $L$, and let $x \in X$ be a point which is not a pole of $s$. Let $\mathfrak{U} \subset \mathfrak{X}$ be an open neighborhood of $\operatorname{red}(x) \in \mathfrak{X}$ on which $\mathfrak{L}$ is trivial. Then $U=\operatorname{red}^{-1}(\mathfrak{U})=\mathfrak{U}_{\eta}$ is a closed analytic domain containing $x$ on which $L$ is trivial, so we can write $\left.s\right|_{U}=f t$, where $t$ is a nonvanishing section of $\left.\mathfrak{L}\right|_{\mathfrak{U}}$ and $f$ is a nonzero meromorphic function on $U$. The formal metric on $L$ induced by $\mathfrak{L}$ is the metric $\|\cdot\|_{\mathfrak{L}}$ defined by

$$
\|s(x)\|_{\mathfrak{L}}:=|f(x)| .
$$

This is independent of all choices because an invertible function on $\mathfrak{U}$ has absolute value 1 everywhere.

In the algebraic situation, let $\mathfrak{X}$ be a proper and flat $R$-scheme with generic fiber $X$, and let $\hat{\mathfrak{X}}$ denote the completion with respect to an ideal of definition in $R$. Then $\hat{\mathfrak{X}}$ is a proper admissible formal $R$-scheme, and there is a canonical isomorphism $X^{\text {an }} \cong \hat{\mathfrak{X}}_{\eta}$. Hence any line bundle $\mathfrak{L}$ on $\mathfrak{X}$ with generic fiber $L$ induces a formal metric $\|\cdot\|_{\mathfrak{L}}$ on $L^{\text {an }}$.

Remark 2.5. Formal metrics have the following intersection-theoretic interpretation over a discretely valued field $K$. (Note that the definition of $\|\cdot\|_{\mathfrak{L}}$ above does not use that $K$ is algebraically closed.) Suppose that $\mathbf{Z}$ is the value group of $K$. For simplicity we restrict ourselves to a regular split semistable model $\mathfrak{X}$ of a smooth, proper, geometrically connected curve $X$. A meromorphic section $s$ of $L$ can be regarded as a meromorphic section of $\mathfrak{L}$, and hence has an order of vanishing $\operatorname{ord}_{D}(s)$ along any irreducible component $D$ of $\mathfrak{X}_{k}$. If $\zeta \in X^{\text {an }}$ is the point reducing to the generic point of $D$, then we have the equality

$$
-\log \|s(\zeta)\|_{\mathfrak{L}}=\operatorname{ord}_{D}(s) .
$$

This follows from the observation that $\operatorname{ord}_{D}: K(X)^{\times} \rightarrow \mathbf{Z}$ reduces to (i.e., is centered at) the generic point of $D$.

Theorem 2.6 (The slope formula). Let $X$ be a smooth, proper, connected $K$-curve, and let $\mathfrak{X}$ be a semistable $R$-model of $X$ with corresponding skeleton $\Gamma_{\mathfrak{X}} \subset X^{\text {an }}$. Assume that $\mathfrak{X}$ is not smooth, so that $\Gamma_{\mathfrak{X}}$ is not a point. Let $\mathfrak{L}$ be a line bundle on $\mathfrak{X}$, let $L=\left.\mathfrak{L}\right|_{X}$, and let 
$s$ be a nonzero meromorphic function on $L$. Let $F=-\left.\log \|s\|_{\mathfrak{L}}\right|_{\Gamma_{\mathfrak{X}}}$. Then $F$ is a tropical meromorphic function on $\Gamma_{\mathfrak{X}}$ and

$$
\tau_{*} \operatorname{div}(s)=\operatorname{div}(F)+\sum_{\zeta} \operatorname{deg}\left(\left.\mathfrak{L}\right|_{D_{\zeta}}\right)(\zeta),
$$

where the sum is taken over vertices $\zeta$ of $\Gamma_{\mathfrak{X}}$ and $D_{\zeta}$ is the irreducible component of $\mathfrak{X}_{k}$ with generic point $\operatorname{red}(\zeta)$.

Proof. If $e \subset \Gamma_{\mathfrak{X}}$ is an open edge, then $\operatorname{red}\left(\tau^{-1}(e)\right)$ is a node in $\mathfrak{X}_{k}$, which is contained in a formal affine open subset of $\hat{\mathfrak{X}}$ on which $\mathfrak{L}$ is trivial. Hence $F=-\log |f|$ on $A=\tau^{-1}(e)$ for some nonzero meromorphic function $f$ on $A$, so $F$ is piecewise affine-linear with integral slopes on $A$ and $\operatorname{div}\left(\left.F\right|_{A}\right)=\tau_{*} \operatorname{div}\left(\left.s\right|_{A}\right)$ by [BPR13, Proposition 2.10(1)]. Since this holds for each edge, $F$ is a tropical meromorphic function on $\Gamma_{\mathfrak{X}}$.

Now let $\zeta$ be a vertex of $\mathfrak{X}$, and let $D=D_{\zeta}$. By blowing up $\mathfrak{X}$ we can add vertices to the interior of loop edges in $\Gamma_{\mathfrak{X}}$. Hence we may assume that $\Gamma_{\mathfrak{X}}$ has no loop edges, so that $D$ is smooth. After multiplying by a nonzero scalar we may also assume that $\|s(\zeta)\|_{\mathfrak{L}}=1$, so that $s$ reduces to a nonzero meromorphic function $\widetilde{s}$ on $D$. Let $\widetilde{x} \in D(k)$, and let $v_{\widetilde{x}}$ be the tangent direction at $\zeta$ in the direction of $\operatorname{red}^{-1}(\widetilde{x})$ (see [BPR13, (5.13)]). Let $\mathfrak{U}$ be an open neighborhood of $\widetilde{x}$ trivializing $\mathfrak{L}$, and let $U=\operatorname{red}^{-1}\left(\mathfrak{U}_{k}\right)$, so $F=-\log |f|$ on $U$ for some nonzero meromorphic function $f$ on $U$ with a well-defined reduction $\widetilde{f}$ on $\mathfrak{U}_{k}$. By [BPR13, Theorem 5.15(3)] we have

$$
\operatorname{ord}_{\widetilde{x}}(\widetilde{s})=\operatorname{ord}_{\widetilde{x}}(\widetilde{f})=d_{v_{\widetilde{x}}} F(\zeta) .
$$

Combining this with [BPR13, Proposition 2.10(2)] yields

$$
\operatorname{ord}_{\widetilde{x}}(\widetilde{s})=\operatorname{deg}\left(\operatorname{div}\left(\left.s\right|_{\operatorname{red}^{-1}(\widetilde{x})}\right)\right)
$$

for all points $\widetilde{x} \in D^{\mathrm{sm}}(k)$, the set of points of $D(k)$ which are not nodes in $\mathfrak{X}_{k}$. Since the edges of $\Gamma_{\mathfrak{X}}$ adjacent to $\zeta$ represent the tangent vectors at $\zeta$ in the direction of the points of $D(k) \backslash D^{\mathrm{sm}}(k)$, we combine the previous two equations to obtain

$$
\begin{aligned}
\operatorname{deg}\left(\left.\mathfrak{L}\right|_{D}\right) & =\sum_{\widetilde{x} \in D(k)} \operatorname{ord}_{\widetilde{x}}(\widetilde{s})=\sum_{\widetilde{x} \in D^{\operatorname{sm}}(k)} \operatorname{ord}_{\widetilde{x}}(\widetilde{s})+\sum_{\widetilde{x} \in D(k) \backslash D^{\operatorname{sm}}(k)} \operatorname{ord}_{\widetilde{x}}(\widetilde{s}) \\
& =\operatorname{deg}\left(\operatorname{div}\left(\left.s\right|_{\tau^{-1}(\zeta)}\right)\right)+\sum_{\widetilde{x} \in D(k) \backslash D^{\operatorname{sm}}(k)} d_{\widetilde{x}_{\widetilde{x}} F(\zeta)} \\
& =\operatorname{deg}\left(\operatorname{div}\left(\left.s\right|_{\tau^{-1}(\zeta)}\right)\right)-\operatorname{ord}_{\zeta}(F) .
\end{aligned}
$$

Equation (2.7) follows.

Remark 2.8. As mentioned above, our slope formula is closely related to the Poincaré-Lelong formula in non-Archimedean Arakelov theory. When the base is a discretely valued field, Theorem 2.6 essentially goes back to Zhang [Zha93]. The term $\sum_{\zeta} \operatorname{deg}\left(\left.\mathfrak{L}\right|_{D_{\zeta}}\right)(\zeta)$ in (2.7) is

\footnotetext{
${ }^{1}$ This theorem is only stated for algebraic meromorphic functions, but is true for analytic meromorphic functions such as $f$ (see [CTT16, Remark 3.6.6]).
} 
precisely the measure $\hat{c}_{1}(\mathfrak{L})$ that Chambert-Loir [CL06] associates to the formally metrized line bundle $L$, where $(\zeta)$ is interpreted as a point mass at $\zeta$. In this language, we have

$$
\hat{c}_{1}(\mathfrak{L})=\operatorname{div}\left(\log \|s\|_{\left.\mathfrak{L}\right|_{\Gamma_{\mathfrak{X}}}}\right)+\tau_{*} \operatorname{div}(s),
$$

where again the divisors are interpreted as counting measures. This is formally similar to the Poincaré-Lelong formula (see [CL11, Lemma 2.2.5] for a precise statement, still over a discretely valued base).

2.4. Integral Rosenlicht differentials. We will apply Theorem 2.6 to sections of a certain canonical extension $\Omega_{\mathfrak{X} / R}^{1}$ of the cotangent bundle $\Omega_{X / K}^{1}$ to our semistable model $\mathfrak{X}$. If $R$ were discretely valued, we could define $\Omega_{\mathfrak{X} / R}^{1}$ as the relative dualizing sheaf, or as the sheaf of logarithmic differentials. In the non-Noetherian case it is easiest to make a somewhat ad-hoc construction, which we develop here as it is nonstandard. To begin we may assume that $K$ is any complete non-Archimedean field with algebraically closed residue field $k$.

Definition 2.9. Let $\mathfrak{X}$ be a (not necessarily proper) semistable $R$-curve with smooth generic fiber, and let $j: \mathfrak{U} \hookrightarrow \mathfrak{X}$ be the inclusion of the smooth locus. The sheaf of integral Rosenlicht differentials on $\mathfrak{X}$ is defined to be

$$
\Omega_{\mathfrak{X} / R}^{1}:=j_{*} \Omega_{\mathfrak{U} / R}^{1},
$$

where $\Omega_{\mathfrak{U} / R}^{1}$ is the usual sheaf of Kähler differentials.

Example 2.10. Let $\mathfrak{X}=\operatorname{Spec}(R[S, T] /(S T-\varpi))$ for some $\varpi \in K^{\times}$with $|\varpi|<1$. The smooth locus $\mathfrak{U}$ is the union of the two distinguished affine open subsets, where $S$ and $T$ are invertible. Hence

$$
H^{0}\left(\mathfrak{X}, \Omega_{\mathfrak{X} / R}^{1}\right)=R\left[S^{ \pm 1}\right] \frac{d S}{S} \cap R\left[T^{ \pm 1}\right] \frac{d T}{T}
$$

inside of $K\left[S^{ \pm 1}\right] d S / S=K\left[T^{ \pm 1}\right] d T / T$. Here we use that $S=\varpi / T$ and $d S / S=-d T / T$. From this it is easy to see that $\Omega_{\mathfrak{X} / R}^{1}$ is a trivial invertible sheaf on $\mathfrak{X}$, with $d S / S=-d T / T$ a nonvanishing section.

Note that a section $\omega \in H^{0}\left(\mathfrak{X}, \Omega_{\mathfrak{X} / R}^{1}\right)$ restricts to a meromorphic section of the cotangent bundle on each component of the special fiber of $\mathfrak{X}$, with at worst a simple pole at the origin, and such that the residues at the origin at each component sum to zero.

Lemma 2.11. Let $\mathfrak{X}$ be a semistable $R$-curve as in Definition 2.9.

(1) The sheaf $\Omega_{\mathfrak{X} / R}^{1}$ is invertible.

(2) If $f: \mathfrak{X}^{\prime} \rightarrow \mathfrak{X}$ is an étale morphism of semistable $R$-curves, then $f^{*} \Omega_{\mathfrak{X} / R}^{1}=\Omega_{\mathfrak{X}^{\prime} / R}^{1}$.

(3) The restriction of $\Omega_{\mathfrak{X} / R}^{1}$ to the special fiber $\mathfrak{X}_{k}$ is isomorphic to the relative dualizing sheaf of $\mathfrak{X}_{k} / k$.

Proof. First we treat (2). Let $j^{\prime}: \mathfrak{U}^{\prime} \hookrightarrow \mathfrak{X}^{\prime}$ be the inclusion of the smooth locus of $\mathfrak{X}^{\prime}$. Then $f^{-1}(\mathfrak{U})=\mathfrak{U}^{\prime}$ and $f^{*} \Omega_{\mathfrak{U} / R}^{1}=\Omega_{\mathfrak{U}^{\prime} / R}^{1}$, so $j_{*}^{\prime} \Omega_{\mathfrak{U}^{\prime} / R}^{1}=f^{*} j_{*} \Omega_{\mathfrak{U} / R}^{1}$ by cohomology and base change for flat morphisms. The first assertion is an immediate consequence of this and Example 2.10, as every singular point of $\mathfrak{X}$ has an étale neighborhood which is étale over $\operatorname{Spec}(R[S, T] /(S T-\varpi))$ for some $\varpi$. 
The Cartesian square

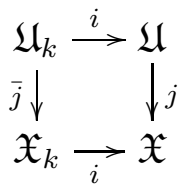

gives rise to a natural homomorphism $\phi: i^{*} \Omega_{\mathfrak{X} / R}^{1} \rightarrow \bar{j}_{*} \Omega_{\mathfrak{U}_{k} / k}^{1}$. By construction this is an isomorphism on $\mathfrak{U}_{k}$. Working étale-locally, it is clear from Example 2.10 that $\phi$ is injective and that its image has the following description. Let $\pi: \widetilde{\mathfrak{X}}_{k} \rightarrow \mathfrak{X}_{k}$ be the normalization. Then a section in the image of $\phi$ in a neighborhood of a singular point $\widetilde{x} \in \mathfrak{X}_{k}$ pulls back to a meromorphic section of $\Omega_{\widetilde{\mathfrak{X}}_{k} / k}^{1}$ with at worst simple poles at the points of $\pi^{-1}(\widetilde{x})$, such that the residues sum to zero. Therefore $i^{*} \Omega_{\mathfrak{U}_{k} / k}^{1}$ is the sheaf of classical Rosenlicht differentials, which is well known to be a dualizing sheaf.

2.4.1. Interpretation in terms of skeletons. Now we suppose that $K$ is algebraically closed and that $\mathfrak{X}$ is a proper semistable $R$-curve with smooth, connected generic fiber $X$. As above, we let $\Gamma_{\mathfrak{X}}$ denote the associated skeleton, considered as a vertex-weighted metric graph.

Lemma 2.12. Let $\zeta \in \Gamma_{\mathfrak{X}}$ be a vertex, and let $D_{\zeta} \subset \mathfrak{X}_{k}$ be the corresponding irreducible component. Then

$$
\operatorname{deg}\left(\left.\Omega_{\mathfrak{X} / R}^{1}\right|_{D_{\zeta}}\right)=2 g(\zeta)-2+\operatorname{deg}(\zeta),
$$

where $g(\zeta)$ is the weight of $\zeta$ and $\operatorname{deg}(\zeta)$ is the valency of $\zeta$ in $\Gamma_{\mathfrak{X}}$.

Proof. This is an immediate consequence of the definitions and Lemma 2.11(3).

The formal metric on $\Omega_{X / K}^{1}$ coming from $\Omega_{\mathfrak{X} / R}^{1}$ can be computed explicitly on $\Gamma_{\mathfrak{X}}$, as follows. Let $e \subset \Gamma_{\mathfrak{X}}$ be an open edge, and let $A=\tau^{-1}(e)$ be an open annulus. Choose an isomorphism $T: A \stackrel{\sim}{\longrightarrow} \mathbf{S}(\varpi)_{+}$with a standard open annulus.

Lemma 2.14. With the above notation, let $\omega=f(T) d T / T$ be the restriction of a nonzero meromorphic section of $\Omega_{X / K}^{1}$ to $A$. Then $\|\omega\|_{\Omega_{X / R}^{1}}=|f|$ on $A$.

Proof. First suppose that $T^{\prime}: A \stackrel{\sim}{\longrightarrow} \mathbf{S}(\varpi)_{+}$is a different isomorphism. A calculation using [BPR13, Proposition 2.2(1)], the explicit description of the units on $\mathbf{S}(\varpi)_{+}$, shows that $d T^{\prime} / T^{\prime}=g(T) d T / T$ for an invertible analytic function $g$ on $\mathbf{S}(\varpi)_{+}$such that $|g(x)|=1$ for all $x \in \mathbf{S}(\varpi)_{+}$. Hence the lemma is true for $T^{\prime}$ if and only if it is true for $T$, so we may choose any parameter $T$ that we like.

Let $\widetilde{x} \in \mathfrak{X}_{k}$ be the nodal point such that $A=\operatorname{red}^{-1}(\widetilde{x})$, let $\phi: \mathfrak{U} \rightarrow \mathfrak{X}$ be an étale neighborhood of $\widetilde{x}$, and let $\widetilde{y} \in \mathfrak{U}$ be an inverse image of $\widetilde{x}$. Then $\phi$ induces an isomorphism $\operatorname{red}^{-1}(\widetilde{y}) \stackrel{\sim}{\longrightarrow} \operatorname{red}^{-1}(\widetilde{x})=A$. Similarly, if $\psi: \mathfrak{U} \rightarrow \operatorname{Spec}(R[S, T] /(S T-\varpi))$ is an étale morphism sending $\widetilde{y}$ to the origin $\widetilde{z}$, then $\psi$ induces an isomorphism $\operatorname{red}^{-1}(\widetilde{y}) \stackrel{\sim}{\longrightarrow} \operatorname{red}^{-1}(\widetilde{z})$. Now the lemma follows from Example 2.10, where it was shown that $d T / T$ is a nonvanishing section of the sheaf of integral Rosenlicht differentials in a neighborhood of $\widetilde{z}$. 
The next lemma says that the restriction of $\|\cdot\|_{\Omega_{\mathfrak{X} / R}^{1}}$ to $\Gamma_{\mathfrak{X}}$ is compatible with refinement of the semistable model giving the skeleton.

Lemma 2.15. Let $\mathfrak{X}, \mathfrak{X}^{\prime}$ be two semistable models of $X$, and suppose that there exists a (necessarily unique) morphism $\mathfrak{X}^{\prime} \rightarrow \mathfrak{X}$ inducing the identity on $X$. Then $\Gamma_{\mathfrak{X}} \subset \Gamma_{\mathfrak{X}^{\prime}}$, and we have $\left.\|\cdot\|_{\Omega_{\mathfrak{X}^{\prime} / R}^{1}}\right|_{\Gamma_{\mathfrak{X}}}=\left.\|\cdot\|_{\Omega_{\mathfrak{X} / R}^{1}}\right|_{\Gamma_{\mathfrak{X}}}$.

Proof. The fact that $\Gamma_{\mathfrak{X}} \subset \Gamma_{\mathfrak{X}^{\prime}}$ follows from [BPR13, Proposition 3.13, Theorem 4.11]. Then $\Gamma_{\mathfrak{X}^{\prime}}$ is obtained from $\Gamma_{\mathfrak{X}}$ by subdividing some edges and adding some new ones. As we are restricting to $\Gamma_{\mathfrak{X}}$, we are not concerned with new edges, so it suffices to show that $\|\cdot\|_{\Omega_{\mathfrak{X} / R}^{1}}$ is insensitive to subdividing an edge, or equivalently, to blowing up a node on $\mathfrak{X}$. But by Lemma 2.14, $\|\cdot\|_{\Omega_{\mathfrak{X} / R}^{1}}$ restricted to an open edge $e$ only depends on a parameter $T$ for $\tau^{-1}(e)$, and $T$ restricts to a parameter on $\tau^{-1}\left(e^{\prime}\right)$ for any $e^{\prime} \subset e$.

By virtue of Lemma 2.15, we will write $\|\cdot\|=\left.\|\cdot\|_{\Omega_{\mathfrak{X} / R}^{1}}\right|_{\Gamma_{\mathfrak{X}}}$ for any semistable model $\mathfrak{X}$.

Remark 2.16. Temkin [Tem14] has developed an extremely general procedure for metrizing the cotangent sheaf on an analytic space, of which the above construction is a special case. However, it is not obvious that the metric resulting from his theory restricts to a formal metric on a skeleton, and therefore one cannot immediately apply Theorem [2.6, as we do in Section 2.4.2.

2.4.2. Interpretation in terms of the canonical divisor of a graph. We assume still that $K$ is algebraically closed and that $\mathfrak{X}$ is a proper semistable $R$-curve with smooth, connected generic fiber $X$. The canonical divisor on $\Gamma_{\mathfrak{X}}$ is by definition

$$
K_{\Gamma_{\mathfrak{X}}}:=\sum(2 g(\zeta)-2+\operatorname{deg}(\zeta))(\zeta),
$$

where the sum is taken over the vertices of $\Gamma_{\mathfrak{X}}$ (see [ABBR15, Definition 2.13]). By Lemma 2.12, equation (2.7) becomes

$$
\tau_{*} \operatorname{div}(\omega)=\operatorname{div}(F)+K_{\Gamma_{\mathfrak{X}}},
$$

where $\omega$ is a nonzero meromorphic 1 -form on $X$, and $F=-\log \|\omega\|$. In particular, if $\omega$ is a regular global 1-form, then

$$
\operatorname{div}(F)+K_{\Gamma_{\mathfrak{X}}} \geq 0
$$

which formally says that $F$ is a "section of the tropical canonical bundle."

2.4.3. Interpretation in terms of intersection theory. Assume for this subsection that $K$ is discretely valued and $\mathfrak{X}$ is semistable, with irreducible decomposition $\mathfrak{X}_{k}=\bigcup C_{i}$ and with dual graph $\Gamma_{\mathfrak{X}}$. Let $\mathfrak{L} \in \operatorname{Pic}(\mathfrak{X})$ be a line bundle, and denote by $\left.\mathfrak{L}\right|_{\Gamma_{\mathfrak{X}}}$ the divisor $\sum\left(\left.\operatorname{deg} \mathfrak{L}\right|_{C_{i}}\right) C_{i} \in \operatorname{Div}\left(\Gamma_{\mathfrak{X}}\right)$. Here we are identifying irreducible components of $\mathfrak{X}_{k}$ with vertices of $\Gamma_{\mathfrak{X}}$. A nonzero regular section $s$ of $\left.\mathfrak{L}\right|_{X}$ extends to a meromorphic section of $\mathfrak{L}$, and after scaling by an element of $K$ extends to a regular section of $\mathfrak{L}$ (with possible zeroes along entire components of $\mathfrak{X}_{k}$ ). 
Write $\operatorname{div}(s)=H+V$, where $H$ is the closure of $\left.\operatorname{div}(s)\right|_{X}$ and $V=\sum n_{i} C_{i}$ is the complement $\operatorname{div}(s)-H$ (so that $\operatorname{Supp}(V) \subset \mathfrak{X}_{k}$ ). Suppose that the support of $H$ is contained in $\mathfrak{X}^{\text {reg}}$; this is guaranteed if $\mathfrak{X}$ is regular and $\left.\operatorname{Supp}(\operatorname{div}(s))\right|_{X} \subset X(K)$. Then $\mathscr{O}(H) \in \operatorname{Pic}(\mathfrak{X})$; if additionally $\mathfrak{X}$ is regular, then $\left.\mathscr{O}(H)\right|_{\Gamma_{\mathfrak{X}}}=\sum \operatorname{deg}\left(C_{i} \cap H\right) C_{i}$.

Let $f: \Gamma_{\mathfrak{X}} \rightarrow \mathbf{Z}$ be given by $f\left(C_{i}\right)=n_{i}$ and extended linearly on edges of $\Gamma_{\mathfrak{X}}$. Then, since $\mathfrak{X}$ is regular, the adjunction formula [Liu02, Theorem 9.1.36] gives

$$
\left.\mathscr{O}(V)\right|_{\Gamma_{\mathfrak{X}}}=-\Delta(f):=\sum_{v} \sum_{e=v w}(f(w)-f(v))(v) .
$$

Since $\mathfrak{L} \cong \mathscr{O}(\operatorname{div}(s)) \cong \mathscr{O}(V) \otimes \mathscr{O}(H)$, this gives the geometric variant of Baker's specialization lemma [Bak08, Lemma 2.8]:

$$
\left.\mathfrak{L}\right|_{\Gamma_{\mathfrak{X}}}+\Delta(f)=\left.\mathscr{O}(H)\right|_{\Gamma_{\mathfrak{X}}} .
$$

When $\mathfrak{L}$ is the relative dualizing sheaf $\omega_{\mathfrak{X} / R}$, this is precisely the "discrete" version of (2.18), as $\left.\omega_{\mathfrak{X} / R}\right|_{\Gamma_{\mathfrak{X}}}=K_{\Gamma_{\mathfrak{X}}}$, and $\Delta(f)$ (resp., $\mathscr{O}(H)_{\Gamma_{\mathfrak{X}}}$ ) plays the role of $\operatorname{div}(F)$ (resp., $\left.\tau_{*} \operatorname{div}(s)\right)$. From the point of view of chip firing, this formula has a more colloquial description: the vanishing of $s$ along components of $\mathfrak{X}_{k}$ gives exactly the firing sequence witnessing the linear equivalence of $\left.\mathfrak{L}\right|_{\Gamma_{\mathfrak{X}}}$ with the divisor $\left.\mathscr{O}(H)\right|_{\Gamma_{\mathfrak{X}}}$ on the graph $\Gamma_{\mathfrak{X}}$.

2.5. Basic wide open subdomains. Assuming now that $K$ is algebraically closed, we return to the notation of Section 2.2. Fix a split semistable $R$-model $\mathfrak{X}$ of $X$ and a type-2 point $\zeta \in \Gamma_{\mathfrak{X}}$. An open star neighborhood of $\zeta$ in $\Gamma_{\mathfrak{X}}$ is a simply connected open neighborhood of the form $V=\{\zeta\} \amalg \bigcup_{i=1}^{r} e_{i}$, where $e_{i}$ is an open interval of length in $\Lambda$ contained in an edge of $\Gamma_{\mathfrak{X}}$ and containing $\zeta$ in its closure, and $r \geq 1$. The inverse image $U=\tau^{-1}(V)$ of an open star neighborhood is called a basic wide open subdomain of $X^{\text {an }}$. The central point of $U$ is $\zeta$ and the underlying affinoid of $U$ is $Y:=\tau^{-1}(\zeta)$. After a suitable blowing up on the special fiber, we can arrange that $\zeta$ is a vertex of $\Gamma_{\mathfrak{X}}$. In this case, if $D_{\zeta} \subset \mathfrak{X}_{k}$ is the irreducible component with generic point $\widetilde{\zeta}=\operatorname{red}(\zeta)$, then $Y=\operatorname{red}^{-1}\left(D_{\zeta}^{\mathrm{sm}}\right)$, where $D_{\zeta}^{\mathrm{sm}}$ is the set of smooth points of $\mathfrak{X}_{k}$ lying on $D_{\zeta}$. Hence

$$
Y \backslash\{\zeta\} \cong \coprod_{\widetilde{x} \in D_{\zeta}^{\mathrm{sm}}} \operatorname{red}^{-1}(\widetilde{x})
$$

where for any smooth point $\widetilde{x} \in \mathfrak{X}_{k}$, the inverse image $\operatorname{red}^{-1}(\widetilde{x})$ is isomorphic to an open unit disc. Moreover, we have $U \backslash Y=\coprod \tau^{-1}\left(e_{i}\right)$, with each $A_{i}:=\tau^{-1}\left(e_{i}\right)$ isomorphic to an open annulus. The closure of $A_{i}$ in $X^{\text {an }}$ is equal to $A_{i} \amalg\left\{\zeta_{i}, \zeta\right\}$, where $\zeta_{i} \in \Gamma_{\mathfrak{X}}$ is the other endpoint of $e_{i}$, which is a type-2 point not contained in $U$. We call $\zeta_{i}$ the end of $U$ associated to $A_{i}$.

Remark 2.20. Our definitions of basic wide open subdomains and their ends are equivalent to those of Coleman [Col89, Section 3] under the identification of a Berkovich analytic space and its corresponding rigid space. More precisely, any basic wide open subdomain of $X^{\text {an }}$ in Coleman's sense is the inverse image of an open star neighborhood of a type-2 point of some skeleton $\Gamma$ of $X$. 


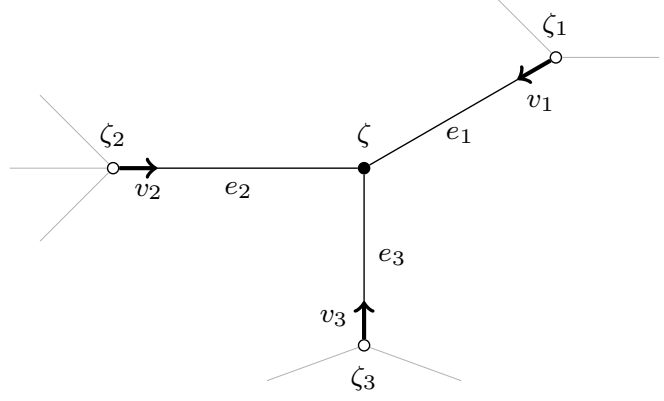

FiguRE 1. An open star neighborhood of a type-2 point $\zeta$ in a skeleton $\Gamma_{\mathfrak{X}}$ and associated notations. The tangent vectors illustrate the statement of Proposition 2.22

Remark 2.21. The open star neighborhood $V$ deformation retracts onto $\zeta$, and the deformation retraction of $X^{\text {an }}$ onto its skeleton retracts $U$ onto $V$. Therefore a basic wide open subdomain is contractible.

We will use the following fundamental relationship between the number of zeros of an analytic function on $U$ and the slopes of its valuation at the ends. This is called the "mass formula" in [BR10, Proposition 5.30], where it is proved for basic wide open subdomains of $\mathbf{P}^{1, \text { an }}$. The situation in the proposition below is illustrated in Figure 1 .

Proposition 2.22. Let $U \subset X^{\text {an }}$ be a basic wide open neighborhood with underlying affinoid $Y$, annuli $A_{1} \amalg \cdots \amalg A_{r}=U \backslash Y$, and corresponding ends $\zeta_{1}, \ldots, \zeta_{r}$. Let $v_{i}$ denote the tangent direction at $\zeta_{i}$ in the direction of $A_{i}$. Let $f$ be a nonzero meromorphic function on $U$ which extends to a meromorphic function on a neighborhood of $\left\{\zeta_{1}, \ldots, \zeta_{r}\right\}$, and let $F=-\log |f|$. Then

$$
\operatorname{deg}\left(\operatorname{div}\left(\left.f\right|_{U}\right)\right)=\sum_{i=1}^{r} d_{v_{i}} F\left(\zeta_{i}\right) .
$$

Proof. This follows from Theorem 2.4 and an easy combinatorial argument.

2.6. de Rham cohomology of a basic wide open. In this section we assume that $K=\mathbf{C}_{p}$ for a prime $p$. We will need Coleman's calculation of the de Rham cohomology of a basic wide open $U=Y \amalg \coprod_{i=1}^{r} A_{i}$. This calculation does not depend on the ambient curve $X$, so by gluing closed discs onto the annuli $A_{i}$ we may assume that $X$ has good reduction and that $U$ is the complement in $X$ of finitely many closed discs contained in distinct residue discs. Let $S \subset X\left(\mathbf{C}_{p}\right) \backslash U\left(\mathbf{C}_{p}\right)$ be a choice of $r$ points, one in each deleted disc.

For a scheme $Z$ over $\mathbf{C}_{p}$, we let $H_{\mathrm{dR}}^{1}(Z)^{\text {alg }}$ denote the algebraic de Rham cohomology of $Z$ over $\mathbf{C}_{p}$, so $H_{\mathrm{dR}}^{1}(X)^{\text {alg }}$ is a $\mathbf{C}_{p}$-vector space of dimension $2 g$, where $g$ is the genus of $X$. We define

$$
H_{\mathrm{dR}}^{1}(U)=\Omega_{U / \mathbf{C}_{p}}^{1}(U) / d \mathscr{O}(U),
$$


the analytic differential forms modulo the exact differentials. Coleman [Col89, Theorem 4.2] proves that the natural restriction map

$$
H_{\mathrm{dR}}^{1}(X \backslash S)^{\text {alg }} \longrightarrow H_{\mathrm{dR}}^{1}(U)
$$

is an isomorphism. In particular,

$$
\operatorname{dim}_{\mathbf{C}_{p}} H_{\mathrm{dR}}^{1}(U)=\operatorname{dim}_{\mathbf{C}_{p}} H_{\mathrm{dR}}^{1}(X \backslash S)^{\mathrm{alg}}=2 g-1+\# S=2 g-1+r,
$$

where $r$ is the number of deleted discs. (The algebraic de Rham cohomology can be calculated using a comparison theorem over $\mathbf{C}$, for example.)

Let $T$ be a parameter on the annulus $A_{i}$, normalized so that $|T(x)| \searrow 1$ as $x \rightarrow \zeta$, the central point of $U$. Let $\omega \in \Omega_{A_{i} / \mathbf{C}_{p}}^{1}\left(A_{i}\right)$, and write

$$
\omega=\sum_{n=-\infty}^{\infty} a_{n} T^{n} \frac{d T}{T} .
$$

The residue of $\omega$ is defined to be $\operatorname{Res}(\omega)=a_{0}$. This is independent of the parameter $T$ up to a sign that is determined by the orientation of the annulus, which we have fixed. Clearly the residue of an exact differential is zero, so Res defines a homomorphism $H_{\mathrm{dR}}^{1}\left(A_{i}\right) \rightarrow \mathbf{C}_{p}$.

Theorem 2.24 (Coleman [Col89, Proposition 4.3, Proposition 4.4]). The following sequence is exact:

$$
0 \longrightarrow H_{\mathrm{dR}}^{1}(X)^{\mathrm{alg}} \longrightarrow H_{\mathrm{dR}}^{1}(U) \stackrel{\oplus \operatorname{Res}}{\longrightarrow} \bigoplus_{i=1}^{r} \mathbf{C}_{p} \stackrel{\sum}{\longrightarrow} \mathbf{C}_{p} \longrightarrow 0
$$

Proof. By [Col89, Proposition 4.4], the sequence is exact at $H_{\mathrm{dR}}^{1}(U)$, so we only need to justify exactness at $\bigoplus_{i=1}^{r} \mathbf{C}_{p}$. Proposition 4.3 in Col89. says that the image is contained in the kernel, so exactness follows from the dimension count (2.23).

\section{INTEGRATION}

We will use two integration theories on curves, namely, Berkovich-Coleman integration and abelian integration. The former is functorial with respect to morphisms and can be calculated by formal antidifferentiation on open annuli. The latter is suitable for use with Chabauty's method. The purpose of this section is to introduce the two integrals and compare them. Related work comparing the two integrals in the context of parallel transport is being undertaken by Besser and Zerbes [BZ].

In this section, we take $K=\mathbf{C}_{p}$, with the valuation normalized so that $\operatorname{val}(p)=1$. We introduce the following notation for a smooth, commutative algebraic or analytic $\mathbf{C}_{p}$-group $G$ and a smooth $\mathbf{C}_{p}$-analytic space $X$ :

$Z_{\mathrm{dR}}^{1}(X)$ The space of closed 1-forms on $X$.

$\Omega_{\text {inv }}^{1}(G) \subset Z_{\mathrm{dR}}^{1}(G)$, the space of invariant 1-forms on $G$.

$\operatorname{Lie}(G) \quad$ The tangent space of $G$ at the identity, dual to $\Omega_{\text {inv }}^{1}(G)$. 
3.1. Integration theories. Let $X$ be a smooth $\mathbf{C}_{p}$-analytic space, and let $\mathcal{P}(X)$ be the set of paths $\gamma:[0,1] \rightarrow X$ with ends in $X\left(\mathbf{C}_{p}\right)$.

Definition 3.1. An integration theory on $X$ is a map $\int: \mathcal{P}(X) \times Z_{\mathrm{dR}}^{1}(X) \rightarrow \mathbf{C}_{p}$ such that:

(1) If $U \subset X$ is an open subdomain isomorphic to an open polydisc and $\left.\omega\right|_{U}=d f$ with $f$ analytic on $U$, then $\int_{\gamma} \omega=f(\gamma(1))-f(\gamma(0))$ for all $\gamma:[0,1] \rightarrow U$.

(2) $\int_{\gamma} \omega$ only depends on the fixed endpoint homotopy class of $\gamma$.

(3) If $\gamma^{\prime} \in \mathcal{P}(X)$ and $\gamma^{\prime}(0)=\gamma(1)$, then

$$
\int_{\gamma^{\prime} * \gamma} \omega=\int_{\gamma} \omega+\int_{\gamma^{\prime}} \omega
$$

(4) $\omega \mapsto \int_{\gamma} \omega$ is linear in $\omega$ for fixed $\gamma$.

Condition (11) completely determines the integration theory on an open polydisc $X$ by the Poincaré lemma: every closed 1-form $\omega \in Z_{\mathrm{dR}}^{1}(X)$ is exact. To be explicit, let $X=$ $\mathbf{B}(1)_{+}$be the 1-dimensional open unit disc. Any $\omega \in Z_{\mathrm{dR}}^{1}\left(\mathbf{B}(1)_{+}\right)$can be written as $\omega=$ $g(T) d T$, where $g(T)=\sum_{n \geq 0} a_{n} T^{n}$ is a convergent power series; then $\omega=d f$, where $f(T)=$ $\sum_{n \geq 0} a_{n} T^{n+1} /(n+1)$ is the power series obtained by formally antidifferentiating $g(T)$. Note that on an open disc, if $g(T)$ is convergent, then $f(T)$ is also convergent. Hence for $\gamma \in \mathcal{P}(X)$ we have $\int_{\gamma} g(T) d T=f(\gamma(1))-f(\gamma(0))$. In higher dimensions one proceeds as above, one variable at a time, as in the proof of the classical Poincaré lemma.

In general, Definition 3.1 does not completely specify an integration theory, because a smooth $\mathbf{C}_{p}$-analytic space, even a smooth proper curve, cannot necessarily be covered (as a Berkovich space) by open polydiscs. The ambiguity is illustrated in the following fundamental example.

Example 3.2. Let $\mathbf{G}_{m}^{\text {an }}$ be the analytification of the multiplicative group over $\mathbf{C}_{p}$ with coordinate $T$. This is a contractible topological space, so any integration theory on $\mathbf{G}_{m}^{\text {an }}$ is by definition path-independent; hence, it makes sense to write $\int_{x}^{y} \omega$ for $x, y \in \mathbf{C}_{p}^{\times}$and $\omega \in Z_{\mathrm{dR}}^{1}\left(\mathbf{G}_{m}^{\mathrm{an}}\right)=H^{0}\left(\mathbf{G}_{m}^{\mathrm{an}}, \Omega_{\mathbf{G}_{m}^{\mathrm{an}} / \mathbf{C}_{p}}^{1}\right)$.

Let $\omega=d T / T$, an invariant 1-form. The formal antiderivative of $\omega$ on the space of "1units" $U_{1}=\left\{x \in \mathbf{G}_{m}^{\text {an }}:|T(x)-1|<1\right\} \cong \mathbf{B}(1)_{+}$is the logarithm given by the usual Mercator series

$$
\log (T)=\sum_{n=1}^{\infty}(-1)^{n+1} \frac{(T-1)^{n}}{n} .
$$

If we require that $x \mapsto \int_{1}^{x} d T / T: \mathbf{C}_{p}^{\times} \rightarrow \mathbf{C}_{p}$ be a group homomorphism, then $\int_{1}^{x} d T / T$ is determined on $\mathscr{O}_{\mathbf{C}_{p}}^{\times}=\left\{x \in \mathbf{C}_{p}^{\times}:|x|=1\right\}$ by the property that $\int_{1}^{x} d T / T=0$ for $x$ a root of unity. Set $\log (x)=\int_{1}^{x} d T / T$ for $x \in \mathscr{O}_{\mathbf{C}_{p}}^{\times}$.

Beyond this, one has to make a choice to specify an integration theory on $\mathbf{G}_{m}^{\text {an }}$. Let $t: \mathbf{Q} \rightarrow \mathbf{C}_{p}^{\times}$be a section of val: $\mathbf{C}_{p}^{\times} \rightarrow \mathbf{Q}$ such that $t(1)=p$, and let $h: \mathbf{Q} \rightarrow \mathbf{C}_{p}$ be any additive group homomorphism. Define $\int_{1}^{x} d T / T=\log \left(x_{1}\right)+h(r)$, where $x=x_{1} \cdot t(r)$ for $r \in \mathbf{Q}$ and $x_{1} \in \mathscr{O}_{\mathbf{C}_{p}}^{\times}$. It turns out that this extends to an integration theory on $\mathbf{G}_{m}^{\text {an }}$ for any 
choice of $h$. (The definition does not depend on $t$, since if $t^{\prime}$ is another section with $t(1)=p$, then $t(r) t^{\prime}(r)^{-1}$ is a root of unity for all $r \in \mathbf{Q}$.)

3.2. Berkovich-Coleman integration. The choice of homomorphism $h: \mathbf{Q} \rightarrow \mathbf{C}_{p}$ in Example 3.2 is the only additional datum necessary for the construction of the BerkovichColeman integration theory. It is equivalent to the following datum.

Definition 3.3. A branch of the logarithm is a homomorphism

$$
\log : \mathbf{C}_{p}^{\times} \longrightarrow \mathbf{C}_{p}
$$

that restricts to $\log$ on $\mathscr{O}_{\mathbf{C}_{p}}^{\times}$.

In the notation of Example 3.2 , we have

$$
\log (x)=\log \left(x_{1}\right)+h(r), \quad \text { where } \quad x=x_{1} \cdot t(r) .
$$

After mandating that the integral be functorial under morphisms of analytic spaces, the integration theory is uniquely specified by equivariance under a lift of Frobenius, a principle attributed to Dwork. This approach to integration has been greatly extended by Berkovich [Ber07]; here, we present only a very small subset of his theory.

Definition 3.5. The Berkovich-Coleman integration theory is an integration theory

$$
\stackrel{\mathrm{BC}}{\mathrm{BC}}(X) \times Z_{\mathrm{dR}}^{1}(X) \longrightarrow \mathbf{C}_{p}
$$

for every smooth $\mathbf{C}_{p}$-analytic space $X$, satisfying:

(1) if $X=\mathbf{G}_{m}^{\text {an }}$, then ${ }^{\mathrm{BC}} \int_{1}^{x} d T / T=\log (x)$, and

(2) if $f: X \rightarrow Y$ is a morphism and $\omega \in Z_{\mathrm{dR}}^{1}(Y)$, then

$$
\int_{\gamma}^{\mathrm{BC}} f^{*} \omega=\int_{f(\gamma)}^{\mathrm{BC}} \omega .
$$

Moreover, condition (1) of Definition 3.1 holds for any open subdomain $U \subset X$.

This integration theory was defined for curves of bad reduction by Coleman and de Shalit CdS88. There, one covers a curve by basic wide open subsets and annuli. A primitive (i.e., an antiderivative) is produced on the basic wide opens by means of Frobenius equivariance and constructed explicitly on annuli by antidifferentiating a power series. This integration theory is closely related to that of Schneider on $p$-adically uniformized curves (see dS06] for details on the comparison).

Example 3.6. Choose $\varpi \in \mathbf{C}_{p}$ with $0<|\varpi|<1$, and let $X=\mathbf{S}(\varpi)_{+}$, the open annulus $|\varpi|<|x|<1$. A closed 1-form $\omega$ can be written

$$
\omega=g(T) \frac{d T}{T}=\sum_{n=-\infty}^{\infty} a_{n} T^{n} \frac{d T}{T}
$$


for a convergent infinite-tailed Laurent series $g(T)$. Let $f(T)=\sum_{n \neq 0}\left(a_{n} / n\right) T^{n}$. Then $d f=\omega-a_{0}(d T / T)$, so for $x, y \in \mathbf{S}(\varpi)\left(\mathbf{C}_{p}\right)$, we have

$$
\int_{x}^{\mathrm{BC}} \omega=\left(f(y)+a_{0} \log (y)\right)-\left(f(x)+a_{0} \log (x)\right) .
$$

Example 3.7. Let $G$ be a smooth, commutative, simply connected $\mathbf{C}_{p}$-analytic group, and let $\omega$ be a (closed) invariant differential on $G$. Since $G$ is simply connected, a BerkovichColeman integral only depends on the endpoints of a path, so it makes sense to write ${ }^{\mathrm{BC}} \int_{1}^{x} \omega$ for $x \in G\left(\mathbf{C}_{p}\right)$. For $x, y \in G\left(\mathbf{C}_{p}\right)$, we have

$$
\int_{1}^{\mathrm{BC}} \omega+\int_{1}^{\mathrm{BC}} \omega=\int_{1}^{\mathrm{BC}} \omega+\int_{x}^{\mathrm{BC}} \omega=\int_{1}^{x y} \omega
$$

where the first equality is by invariance of $\omega$ and the second is by Definition 3.1](3). Therefore $x \mapsto{ }^{\mathrm{BC}} \int_{1}^{x} \omega$ is a group homomorphism $G\left(\mathbf{C}_{p}\right) \rightarrow \mathbf{C}_{p}$.

In what follows, we will pick once and for all a branch of logarithm. A convenient choice is $\log (p)=0$; that is, $h=0$.

3.3. The abelian integral. Another approach to defining a $p$-adic integration theory on a curve is via $p$-adic Lie theory on its Jacobian. This was done in great generality by Zarhin [Zar96]. This method was extended to the $p$-adic Tate module of abelian varieties by Colmez [Col92]. Other references for this integration theory are [Bre00], Vol03] and the second part of [CI99], taken with the understanding that the first part uses the BerkovichColeman integration theory.

Recall that if $A$ is an abelian variety over $K$, then

$$
\Omega_{A / K}^{1}(A)=\Omega_{\mathrm{inv}}^{1}(A)=Z_{\mathrm{dR}}^{1}(A)
$$

because all global 1-forms on a proper group scheme are invariant, and any invariant 1-form on a smooth, commutative algebraic group is closed.

Definition 3.8. Let $A$ be an abelian variety over $\mathbf{C}_{p}$. The abelian logarithm on $A$ is the unique homomorphism of $\mathbf{C}_{p}$-Lie groups $\log _{A\left(\mathbf{C}_{p}\right)}: A\left(\mathbf{C}_{p}\right) \rightarrow \operatorname{Lie}(A)$ such that

$$
d \log _{A\left(\mathbf{C}_{p}\right)}: \operatorname{Lie}(A) \longrightarrow \operatorname{Lie}(\operatorname{Lie}(A))=\operatorname{Lie}(A)
$$

is the identity map.

See [Zar96] for the existence and uniqueness of $\log _{A\left(\mathbf{C}_{p}\right)}$. For $x \in A\left(\mathbf{C}_{p}\right)$ and $\omega \in \Omega_{A / \mathbf{C}_{p}}^{1}(A)$, we define

$$
\int_{0}^{\mathrm{Ab}} \omega=\left\langle\log _{A\left(\mathbf{C}_{p}\right)}(x), \omega\right\rangle,
$$

where $\langle\cdot, \cdot\rangle$ is the pairing between $\operatorname{Lie}(A)$ and $\Omega_{A / \mathbf{C}_{p}}^{1}(A)$. For $x, y \in A\left(\mathbf{C}_{p}\right)$, we set

$$
\int_{y}^{\mathrm{Ab}} \omega=\int_{0}^{\mathrm{Ab}} \omega-\int_{0}^{\mathrm{Ab}} \omega .
$$

We call ${ }^{\mathrm{Ab}} \int$ the abelian integral on $A$. 
The abelian logarithm and the abelian integral are functorial under homomorphisms of abelian varieties: if $f: A \rightarrow B$ is a homomorphism, then $d f \circ \log _{A\left(\mathbf{C}_{p}\right)}=\log _{B\left(\mathbf{C}_{p}\right)} \circ f$ and

$$
\int_{\gamma}^{\mathrm{Ab}} f^{*} \omega=\int_{f(\gamma)}^{\mathrm{Ab}} \omega
$$

for $\omega \in \Omega_{B / \mathbf{C}_{p}}^{1}(B)$.

Proposition 3.10. The abelian integral $(\gamma, \omega) \mapsto{ }^{\mathrm{Ab}} \int_{\gamma(0)}^{\gamma(1)} \omega$ is an integration theory on $A^{\mathrm{an}}$ in the sense of Definition 3.1.

We postpone the proof until after the comparison result, which is Proposition 3.16.

3.4. Comparison between the Berkovich-Coleman and abelian integrals. Before comparing the two integration theories, we consider the following motivating example (see also [CI99, Ex. 7.4]).

Example 3.11. Let $E$ be an elliptic curve over $\mathbf{C}_{p}$ with bad reduction. Then $E$ is a Tate curve; that is, it has a uniformization $E^{\text {an }} \cong \mathbf{G}_{m}^{\text {an }} / q^{\mathbf{Z}}$ for a unique value $q \in \mathbf{C}_{p}$ with $0<|q|<1$. As $\mathbf{G}_{m}^{\text {an }}$ is contractible, the projection $\pi: \mathbf{G}_{m}^{\text {an }} \rightarrow E^{\text {an }}$ is a universal covering space (in the sense of point-set topology), with deck transformation group $q^{\mathbf{Z}}$.

Let $\omega$ be the invariant 1-form on $E$ which pulls back to $d T / T$ on $\mathbf{G}_{m}^{\text {an }}$. Let $\gamma:[0,1] \rightarrow E^{\text {an }}$ be a path with $\gamma(0)=0$ and $\gamma(1)=x \in E\left(\mathbf{C}_{p}\right)$, let $\widetilde{\gamma}:[0,1] \rightarrow \mathbf{G}_{m}^{\text {an }}$ be the unique lift of $\gamma$ with $\widetilde{\gamma}(0)=1$, and let $\widetilde{x}=\widetilde{\gamma}(1)$. By the definition and the functoriality of the BerkovichColeman integral, we have

$$
\int_{\gamma} \omega=\int_{\widetilde{\gamma}}^{\mathrm{BC}} \frac{d T}{T}=\log (\widetilde{x}) .
$$

On the other hand, the abelian integral gives rise to a (potentially) different branch of the logarithm $\log _{\mathrm{Ab}}: \mathbf{C}_{p}^{\times} \rightarrow \mathbf{C}_{p}$ by setting

$$
\log _{\mathrm{Ab}}(\widetilde{x}):=\int_{0}^{\mathrm{Ab}} \omega=\int_{\gamma} \omega .
$$

This branch of the logarithm $\log _{\mathrm{Ab}}$ comes from the homomorphism $h_{\mathrm{Ab}}: \mathbf{Q} \rightarrow \mathbf{C}_{p}$ defined by Q-linearity and $h_{\mathrm{Ab}}(\operatorname{val}(q))=\log (t(\operatorname{val}(q)) / q)$. As both $\log$ and $\log _{\mathrm{Ab}}$ restrict to log on $\mathscr{O}_{\mathbf{C}_{p}}^{\times}$, their difference $\log -\log _{\mathrm{Ab}}$ descends to a homomorphism from $\mathbf{C}_{p}^{\times} / \mathscr{O}_{\mathbf{C}_{p}}^{\times}=\mathbf{Q}$ to $\mathbf{C}_{p}$, and we have

$$
\int_{\gamma}^{\mathrm{BC}} \omega-{ }^{\mathrm{Ab}} \int_{\gamma} \omega=\log (\widetilde{x})-\log _{\mathrm{Ab}}(\widetilde{x})=\left(h-h_{\mathrm{Ab}}\right)(\operatorname{val}(\widetilde{x})) .
$$

In particular, the difference between the integrals is a Q-linear function of the valuation of $\widetilde{x}$. We will show that this fact, suitably interpreted, holds in general. 
3.4.1. Non-Archimedean uniformization of abelian varieties. To study the general situation we will make use of the non-Archimedean analytic uniformization of abelian varieties, in Berkovich's language. The canonical references are [BL84] and [BL91] (see also [BR14, Section 4] for a summary).

Let $A$ be an abelian variety over $\mathbf{C}_{p}$, and let $\pi: E^{\text {an }} \rightarrow A^{\text {an }}$ be the topological universal cover of $A^{\text {an }}$. Then $E^{\text {an }}$ has the unique structure of a $\mathbf{C}_{p}$-analytic group (after choosing an identity element), and the kernel $M^{\prime}$ of $\pi$ is canonically isomorphic to $\pi_{1}\left(A^{\text {an }}\right)=H_{1}\left(A^{\text {an }}, \mathbf{Z}\right)$. Moreover, $E^{\text {an }}$ is the analytification of an algebraic $\mathbf{C}_{p}$-group $E$, which is an extension of an abelian variety $B$ with good reduction by a torus $T$. This uniformization theory is summarized in the Raynaud uniformization cross:

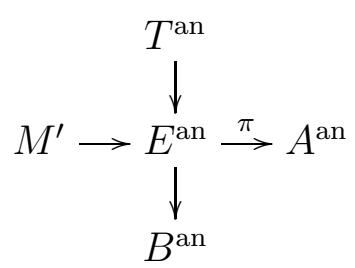

Let $M$ be the character lattice of $T$, so $T=\operatorname{Spec}\left(\mathbf{C}_{p}[M]\right)$. The abelian variety $A$ has a semiabelian $\mathscr{O}_{\mathbf{C}_{p}}$-model $\mathscr{A}$ whose special fiber $\bar{A}$ fits into the short exact sequence

$$
0 \longrightarrow \bar{T} \longrightarrow \bar{A} \longrightarrow \bar{B} \longrightarrow 0
$$

where $\bar{T}=\operatorname{Spec}\left(\overline{\mathbf{F}}_{p}[M]\right)$ and $\bar{B}$ is the reduction of $B$. Let $\hat{A}$ be the $p$-adic completion of $\mathscr{A}$, and let $A_{0}=\hat{\mathscr{A}}_{\eta}$ be its analytic generic fiber. This is an analytic domain subgroup in $A^{\text {an }}$ which lifts in a unique way to an analytic domain subgroup in $E^{\text {an }}$. It fits into the short exact sequence

$$
0 \longrightarrow T_{0} \longrightarrow A_{0} \longrightarrow B^{\text {an }} \longrightarrow 0
$$

where $T_{0}=\mathscr{M}\left(\mathbf{C}_{p}\langle M\rangle\right)$ is the affinoid torus inside of $T^{\text {an }}$.

Let $N=\operatorname{Hom}(M, \mathbf{Z})$ be the dual of the character lattice of $T$, with $(\cdot, \cdot): N \times M \rightarrow \mathbf{Z}$ the evaluation pairing. For $u \in M$, we let $\chi^{u} \in \mathbf{C}_{p}[M]$ denote the corresponding character of $T$. We have a tropicalization map trop: $T^{\text {an }} \rightarrow N_{\mathbf{R}}=\operatorname{Hom}(M, \mathbf{R})$ defined by $(\operatorname{trop}(\|\cdot\|), u)=$ $-\log \left\|\chi^{u}\right\|$, where we regard $T^{\text {an }}$ as a space of seminorms on $\mathbf{C}_{p}[M]$. The map trop is surjective, continuous, and proper, and the affinoid torus $T_{0}$ is equal to $\operatorname{trop}^{-1}(0)$. We extend trop to all of $E^{\text {an }}$ by declaring that $\operatorname{trop}^{-1}(0)=A_{0}$. We have $\operatorname{trop}\left(E\left(\mathbf{C}_{p}\right)\right)=N_{\mathbf{Q}}=$ $\operatorname{Hom}(M, \mathbf{Q})$, so the map trop: $E\left(\mathbf{C}_{p}\right) \rightarrow N_{\mathbf{Q}}$ is a surjective group homomorphism with kernel $A_{0}\left(\mathbf{C}_{p}\right)$. The restriction of trop to $M^{\prime} \subset E\left(\mathbf{C}_{p}\right)$ is injective, and its image $\operatorname{trop}\left(M^{\prime}\right) \subset N_{\mathbf{Q}}$ is a full-rank lattice in the real vector space $N_{\mathbf{R}}$. Let $\Sigma=\Sigma(A)$ be the real torus $N_{\mathbf{R}} / \operatorname{trop}(M)$. Since $A^{\text {an }}$ is the quotient of $E^{\text {an }}$ by the action of $M^{\prime}$, there exists a unique map $\bar{\tau}: A^{\text {an }} \rightarrow \Sigma$ 
making the following exact diagram commute:

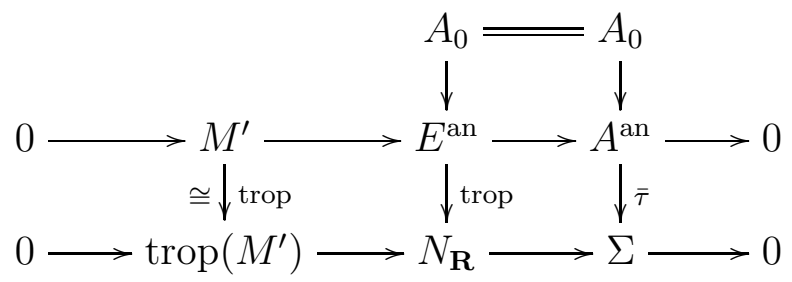

The real torus $\Sigma$ is called the skeleton of $A$; in fact there exists a canonical section $\Sigma \rightarrow$ $A^{\text {an }}$ of $\bar{\tau}$, and $A^{\text {an }}$ deformation retracts onto its image [Ber90, Section 6.5]. Letting $\Sigma_{\mathbf{Q}}=$ $N_{\mathbf{Q}} / \operatorname{trop}\left(M^{\prime}\right)$ and taking $\mathbf{C}_{p}$-points, we have a surjective homomorphism of short exact sequences

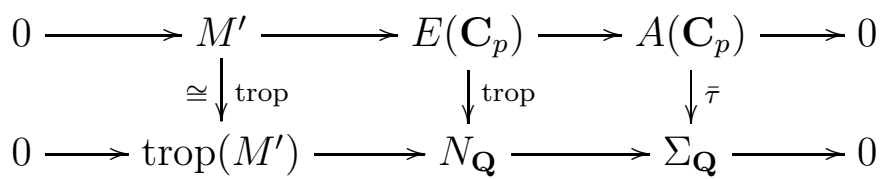

where $A_{0}\left(\mathbf{C}_{p}\right)$ is the kernel of the middle and right vertical arrows.

3.4.2. Comparison of the integrals. Since $E^{\text {an }}$ is locally isomorphic to $A^{\text {an }}$, or since any invariant 1-form on $E^{\text {an }}$ descends to an invariant 1-form on $A^{\text {an }}$, we have canonical identifications

$$
\operatorname{Lie}(E)=\operatorname{Lie}(A) \quad \text { and } \quad \Omega_{\text {inv }}^{1}(E)=\Omega_{A / \mathbf{C}_{p}}^{1}(A) .
$$

Since $E^{\text {an }}$ is simply connected, by Example 3.7 we may define a homomorphism

$$
\log _{\mathrm{BC}}: E\left(\mathbf{C}_{p}\right) \longrightarrow \operatorname{Lie}(A), \quad x \mapsto \int_{0}^{\mathrm{BC}}
$$

Composing the abelian logarithm $\log _{A\left(\mathbf{C}_{p}\right)}: A\left(\mathbf{C}_{p}\right) \rightarrow \operatorname{Lie}(A)$ with $\pi: E^{\text {an }} \rightarrow A^{\text {an }}$ yields

$$
\log _{\mathrm{Ab}}: E\left(\mathbf{C}_{p}\right) \longrightarrow \operatorname{Lie}(A), \quad x \mapsto \int_{0}^{\mathrm{Ab}}
$$

Proposition 3.16. The difference

$$
\log _{\mathrm{BC}}-\log _{\mathrm{Ab}}: E\left(\mathbf{C}_{p}\right) \longrightarrow \operatorname{Lie}(A)
$$

between the two logarithms factors as

$$
E\left(\mathbf{C}_{p}\right) \stackrel{\text { trop }}{\longrightarrow} N_{\mathbf{Q}} \stackrel{L}{\longrightarrow} \operatorname{Lie}(A),
$$

where $L$ is Q-linear.

We have the following interpretation of Proposition 3.16 in terms of paths. Let $\gamma:[0,1] \rightarrow$ $A^{\text {an }}$ be a path with $\gamma(0)=0$ and $\gamma(1)=x \in A\left(\mathbf{C}_{p}\right)$. Let $\widetilde{\gamma}:[0,1] \rightarrow E^{\text {an }}$ be the unique lift starting at 0 , and let $\widetilde{x}=\widetilde{\gamma}(1) \in E\left(\mathbf{C}_{p}\right)$. Then for $\omega \in \Omega_{A / \mathbf{C}_{p}}^{1}(A)$, we have

$$
\int_{\gamma}^{\mathrm{BC}} \omega-\int_{\gamma}^{\mathrm{Ab}} \omega=\langle L \circ \operatorname{trop}(\widetilde{x}), \omega\rangle,
$$


where $\langle\cdot, \cdot\rangle$ denotes the pairing between $\operatorname{Lie}(A)$ and $\Omega_{A / \mathbf{C}_{p}}^{1}(A)$. Because $\log _{\mathrm{Ab}}$ vanishes on $\operatorname{trop}\left(M^{\prime}\right) \subset E\left(\mathbf{C}_{p}\right)$, the homomorphism $L$ is uniquely determined by

$$
\langle L \circ \operatorname{trop}(\widetilde{x}), \omega\rangle=\int_{\gamma}^{\mathrm{BC}} \omega \quad \text { for } \quad \gamma \in \pi_{1}\left(A^{\mathrm{an}}, 0\right) .
$$

Proof of Proposition 3.16. Since $A_{0}\left(\mathbf{C}_{p}\right)=\operatorname{ker}\left(\operatorname{trop}: E\left(\mathbf{C}_{p}\right) \rightarrow N_{\mathbf{Q}}\right)$, the proof amounts to showing that $\log _{\mathrm{BC}}=\log _{\mathrm{Ab}}$ on $A_{0}\left(\mathbf{C}_{p}\right)$. According to [Zar96], the abelian integral on an abelian $\mathbf{C}_{p}$-Lie group $G$ exists and is characterized by (3.9) whenever $G$ has the property that $G / U$ is a torsion group for all open subgroups $U$ of $G$. This property is satisfied by $A\left(\mathbf{C}_{p}\right)$. Since $A_{0}\left(\mathbf{C}_{p}\right)$ is an analytic domain in $A\left(\mathbf{C}_{p}\right)$, it is an open subgroup of $A\left(\mathbf{C}_{p}\right)$ in the naïve analytic topology, so the property is also satisfied by $A_{0}\left(\mathbf{C}_{p}\right)$. Hence $\left.\log _{\mathrm{Ab}}\right|_{A_{0}\left(\mathbf{C}_{p}\right)}$ is characterized by the fact that it induces the identity map on tangent spaces.

On the other hand, $A_{0}$ is simply connected - the deformation retraction of $A^{\text {an }}$ onto $\Sigma$ takes $A_{0}$ onto $\{0\}$ - so the Berkovich-Coleman integral on $A_{0}\left(\mathbf{C}_{p}\right)$ is path-independent. Hence it suffices to show that $\log _{\mathrm{BC}}$ induces the identity map on $\operatorname{Lie}\left(A_{0}\right)=\operatorname{Lie}(A)$. But $0 \in A$ has a neighborhood $U$ isomorphic to an open unit polydisc, so $\log _{\mathrm{BC}}$ can be calculated on $U$ by formal antidifferentiation as in Section 3.1.

Because $N=0$ for abelian varieties of good reduction, we have the following.

Corollary 3.18. The Berkovich-Coleman and abelian integrals coincide on abelian varieties of good reduction.

Remark 3.19. Given a branch of the logarithm, Zarhin [Zar96] defines an abelian integration theory for any commutative $\mathbf{C}_{p}$-algebraic group $G$. The proof of Proposition 3.16 shows that the Berkovich-Coleman integral coincides with Zarhin's integral on any $G$ such that $G^{\text {an }}$ is simply connected and admits a neighborhood of 1 which is isomorphic to a unit polydisc.

Proof of Proposition 3.10. The only part of Definition 3.1 that does not follow immediately from the definitions is condition (1), the fundamental theorem of calculus on open polydiscs. Let $U \subset A^{\text {an }}$ be an open subdomain isomorphic to an open polydisc. As $U$ is simply connected, it lifts to an open subdomain $\widetilde{U} \subset E^{\text {an }}$ which maps isomorphically onto $U$. By Proposition 3.16, it suffices to show that $\operatorname{trop}(\widetilde{U})$ is a single point.

Choosing a basis for $N$, we can think of trop as a map $E^{\text {an }} \rightarrow \mathbf{R}^{n}$. As explained in the paragraph after the statement in [BL91, Theorem 1.2], the extensions $0 \rightarrow T_{0} \rightarrow A_{0} \rightarrow$ $B^{\text {an }} \rightarrow 0$ and $0 \rightarrow T \rightarrow E^{\text {an }} \rightarrow B^{\text {an }} \rightarrow 0$ split locally on $B^{\text {an }}$ in a compatible way. It follows that the coordinates of trop locally (on $B^{\text {an }}$ ) have the form $-\log |f|$ for $f$ an invertible function. Therefore the claim is a consequence of Lemma 3.20 below.

Lemma 3.20. Let $F: \mathbf{B}^{n}(1)_{+} \rightarrow \mathbf{R}$ be a continuous function which locally has the form $F(x)=-\log |f(x)|$ for an invertible function $f$. Then $F$ is constant.

Proof. As $\mathbf{B}^{n}(1)_{+}$is covered by closed polydiscs of smaller radius, it suffices to prove the lemma for the closed polydisc $\mathbf{B}^{n}(1)$ instead. First we prove the lemma when $n=1$. Since $F$ is locally of the form - $\log |f|$, it is harmonic in the sense of [BPR13, Definition 5.14]: 
this follows from the slope formula [BPR13, Theorem 5.15] and the fact that harmonicity is a local condition. Therefore the mean value theorem applies, so $F$ attains its maximum on the Shilov boundary point $\zeta$ of $\mathbf{B}(1)$. By the same reasoning as applied to $-F$, we see that $F$ also attains its minimum on $\zeta$. Thus $F$ is constant.

The general case follows from the above and these observations: (a) any two $\mathbf{C}_{p}$-points of $\mathbf{B}^{n}(1)$ are in the image of a morphism $\mathbf{B}(1) \rightarrow \mathbf{B}^{n}(1)$, and (b) the $\mathbf{C}_{p}$-points of $\mathbf{B}^{n}(1)$ are dense in $\mathbf{B}^{n}(1)$ by [Ber90, Proposition 2.1.15].

We extract the following statement from the proof of Proposition 3.10 .

Proposition 3.21. Let $\phi: \mathbf{B}^{n}(1)_{+} \rightarrow E^{\text {an }}$ be a morphism. Then trop $\circ \phi: \mathbf{B}^{n}(1)_{+} \rightarrow N_{\mathbf{R}}$ is constant.

3.5. Integration on curves of any reduction type. Fix a smooth, proper, connected $\mathbf{C}_{p}$-curve $X$ of genus at least 1 , let $J$ be its Jacobian, and let $\iota: X \hookrightarrow J$ be the Abel-Jacobi map defined with respect to a choice of basepoint $x_{0} \in X\left(\mathbf{C}_{p}\right)$. Note that $\iota^{*}: \Omega_{J / \mathbf{C}_{p}}^{1}(J) \rightarrow$ $\Omega_{X / \mathbf{C}_{p}}^{1}(X)$ is an isomorphism which does not depend on the choice of $x_{0}$.

As $X^{\text {an }}$ is a smooth analytic space, it has a Berkovich-Coleman integration theory ${ }^{\mathrm{BC}}$ as explained in Section 3.2 .

Definition 3.22. The abelian integral on $X^{\mathrm{an}}$ is the map ${ }^{\mathrm{Ab}} \int: \mathcal{P} \times \Omega_{X / \mathbf{C}_{p}}^{1}(X) \rightarrow \mathbf{C}_{p}$ defined by

$$
\int_{\gamma}^{\mathrm{Ab}} \iota^{*} \omega=\int_{\iota \circ \gamma}^{\mathrm{Ab}} \omega .
$$

See [Zar96] for a much more general construction along these lines.

Lemma 3.23. The abelian integral is an integration theory on $X^{\text {an }}$ in the sense of Definition 3.1, which is independent of the choice of basepoint $x_{0}$.

Proof. The only statement that does not follow immediately from the definitions is condition (11), which is a consequence of Proposition 3.21.

By Corollary 3.18, the Berkovich-Coleman and abelian integrals coincide on $X^{\text {an }}$ when $J$ has good reduction and, in particular, when $X$ has good reduction. In this rest of this section, we will make explicit the difference between the Berkovich-Coleman and abelian integrals on a basic wide open subdomain in terms of the tropical Abel--Jacobi map.

3.5.1. The tropical and algebraic Abel-Jacobi maps. Let $\Gamma \subset X^{\text {an }}$ be a skeleton of $X$ with retraction map $\tau: X^{\text {an }} \rightarrow \Gamma$, as in Section 2.2. The Jacobian of the metric graph $\Gamma$ is the quotient $J(\Gamma)=\operatorname{Div}^{0}(\Gamma) / \operatorname{Prin}(\Gamma)$, where $\operatorname{Div}^{0}(\Gamma)$ is the group of degree-zero divisors in $\Gamma$ and $\operatorname{Prin}(\Gamma)$ is the subgroup of divisors of meromorphic functions on $\Gamma$ (see Section 2.3). The Jacobian of $\Gamma$ is a real torus and is moreover a principally polarized tropical abelian variety in the sense of [BR14, Section 3.7]. Fixing a basepoint $P_{0} \in \Gamma$, we define the tropical Abel-Jacobi map $\beta: \Gamma \rightarrow J(\Gamma)$ by the usual formula

$$
\left.\beta(P)=\underset{24}{[(P)}-\left(P_{0}\right)\right] .
$$


Let $\Sigma=N_{\mathbf{R}} / \operatorname{trop}\left(M^{\prime}\right)$ be the skeleton of $J^{\text {an }}$, and let $\bar{\tau}: J^{\text {an }} \rightarrow \Sigma$ be the retraction map, as in Section 3.4.1.

Theorem 3.24 (Baker and Rabinoff [BR14, Theorem 2.9, Proposition 5.3]). There is a canonical isomorphism $\Sigma \stackrel{\sim}{\longrightarrow} J(\Gamma)$ making the following square commute:

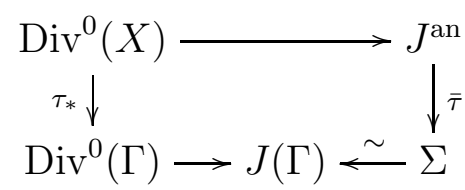

In other words, for $D \in \operatorname{Div}^{0}(X)$, the point $\bar{\tau}([D]) \in \Sigma$ is identified with $\left[\tau_{*} D\right] \in J(\Gamma)$.

From now on we will implicitly identify $J(\Gamma)$ with $\Sigma$. In [BR14, Proposition 6.2] it is shown that Theorem 3.24 is compatible (under retraction) with the algebraic and tropical Abel-Jacobi maps.

Proposition 3.25 (Baker and Rabinoff [BR14, Proposition 6.2]). Fix $x_{0} \in X\left(\mathbf{C}_{p}\right)$ and $P_{0}=\tau\left(x_{0}\right) \in \Gamma$, and let $\iota: X \rightarrow J$ and $\beta: \Gamma \rightarrow \Sigma$ be the corresponding Abel-Jacobi maps. Then the following square commutes:

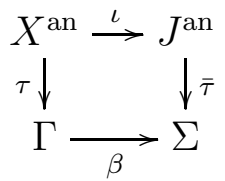

From now on we assume that the algebraic and tropical Abel-Jacobi maps are taken with respect to compatible basepoints as in Proposition 3.25, Let $V \subset \Gamma$ be a simply connected open subgraph with edge lengths in $\mathbf{Q}$, and let $U=\tau^{-1}(V)$, an open analytic domain in $X^{\text {an }}$. For example, $U$ could be a basic wide open subdomain. As $U$ is simply connected as well, the restriction of the Abel-Jacobi map $\iota: X^{\text {an }} \rightarrow J^{\text {an }}$ to $U$ lifts uniquely through the universal cover $\pi: E^{\text {an }} \rightarrow J^{\text {an }}$ to a morphism $\widetilde{\iota}: U \rightarrow E^{\text {an }}$ taking the basepoint to the origin. Since $U \backslash V$ is a disjoint union of open discs, each retracting to a unique point of $V$, by Proposition 3.21 the composition trop $\circ \widetilde{\iota}: U \rightarrow N_{\mathbf{R}}$ factors through the retraction to the skeleton $\tau: U \rightarrow V$. Moreover, by Proposition 3.25 the restriction $\widetilde{\beta}$ of trop $\circ \widetilde{\iota}$ to $V$ is a lift of the restriction of the tropical Abel-Jacobi map $\beta: \Gamma \rightarrow \Sigma$ to $V$ through the universal covering map $N_{\mathbf{R}} \rightarrow \Sigma$. In summary, the following diagram is commutative:

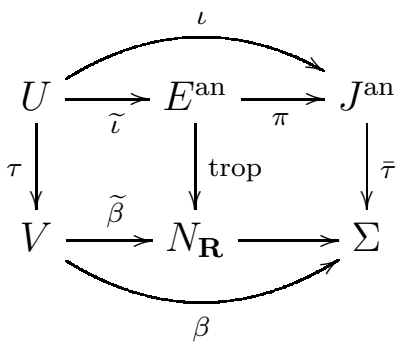

The following result of Mikhalkin and Zharkov, which is a consequence of the discussion in Section 6 of [MZ08] (see also [BF11, Theorem 4.1]), says that the map $\widetilde{\beta}: V \rightarrow N_{\mathbf{R}}$ is very well behaved. 
Theorem 3.27 (Mikhalkin and Zharkov [MZ08, Section 6]). The partial lift $\widetilde{\beta}: V \rightarrow N_{\mathbf{R}}$ of $\beta: \Gamma \rightarrow \Sigma$ satisfies the following properties.

(1) If $e \subset V$ is an edge such that $\Gamma \backslash e$ is disconnected, then $\widetilde{\beta}$ is constant on $e$.

(2) If $e \subset V$ is an edge such that $\Gamma \backslash e$ is connected, then $\widetilde{\beta}$ is affine-linear on $e$ with rational slopes.

(3) Vertices of $V$ map into $N_{\mathbf{Q}}$.

(4) $\widetilde{\beta}$ satisfies the tropical balancing condition.

The balancing condition in the last part of Theorem 3.27 roughly says that at any vertex $v \in V$, a weighted sum of the images of the tangent vectors at $v$ under $\widetilde{\beta}$ is equal to zero. This implies, for instance, that if $v$ has three adjacent edges $e_{1}, e_{2}, e_{3}$, then their images under $\widetilde{\beta}$ are coplanar (see the end of [BF11, Section 3] for details).

3.5.2. Comparison of the integrals, bis. Now we are able to draw some consequences for integration on basic wide open subdomains. Suppose that $V$ is an open star neighborhood of a type-2 point $\zeta \in \Gamma$ as in Section 2.5, so that $U=\tau^{-1}(V)$ is a basic wide open subdomain. Recall that $\operatorname{deg}(\zeta)$ denotes the valency of $\zeta$ as a vertex in $\Gamma$, which is at least 1 since a basic wide open by definition has at least one end.

Proposition 3.28. Let $H \subset \Omega_{X / \mathbf{C}_{p}}^{1}(X)$ be the subspace of those 1 -forms $\omega$ such that ${ }^{\mathrm{BC}} \int_{\gamma} \omega=$ ${ }^{\mathrm{Ab}} \int_{\gamma} \omega$ for all paths $\gamma:[0,1] \rightarrow U$ with endpoints in $U\left(\mathbf{C}_{p}\right)$. Then the codimension of $H$ in $\Omega_{X / \mathbf{C}_{p}}^{1}(X)$ is strictly less than $\operatorname{deg}(\zeta)$.

Proof. We are free to choose the basepoint $x_{0}$ in $U\left(\mathbf{C}_{p}\right)$ such that $P_{0}=\tau\left(x_{0}\right)=\zeta$. We choose the lift $\widetilde{\iota}: U \rightarrow E^{\text {an }}$ of $\iota$ such that $\widetilde{\iota}\left(x_{0}\right)=0$. Since we can compose paths, we have $\omega \in H$ if and only if ${ }^{\mathrm{BC}} \int_{\gamma} \omega={ }^{\mathrm{Ab}} \int_{\gamma} \omega$ for all paths $\gamma$ such that $\gamma(0)=x_{0}$. As $U$ is simply connected, the Berkovich-Coleman integral is path-independent, so we write ${ }^{\mathrm{BC}} \int_{x_{0}}^{x} \omega={ }^{\mathrm{BC}} \int_{\gamma} \omega$ for any path $\gamma$ from $x_{0}$ to $x \in U\left(\mathbf{C}_{p}\right)$.

By Proposition 3.16 and (3.17), there is a linear map

$$
L: N_{\mathbf{Q}} \rightarrow \operatorname{Lie}(J)=\operatorname{Hom}\left(\Omega_{X / \mathbf{C}_{p}}^{1}(X), \mathbf{C}_{p}\right)
$$

such that for all $\omega \in \Omega_{X / \mathbf{C}_{p}}^{1}(X)$ and all $x \in U\left(\mathbf{C}_{p}\right)$, we have

$$
\int_{x_{0}}^{\mathrm{BC}} \omega-\int_{x_{0}}^{x} \omega=\langle L \circ \operatorname{trop}(\widetilde{\iota}(x)), \omega\rangle .
$$

By the balancing condition in Theorem 3.27, $\operatorname{trop}\left(\widetilde{\iota}\left(U\left(\mathbf{C}_{p}\right)\right)\right)=\widetilde{\beta}(V) \cap N_{\mathbf{Q}}$ spans a Q-vector space of dimension at most $\operatorname{deg}(\zeta)-1$ (note that $\widetilde{\beta}(\zeta)=0$ since $\zeta$ is the basepoint of the tropical Abel-Jacobi map $\beta)$. Therefore the annihilator $H$ of $L\left(\operatorname{trop}\left(\widetilde{\iota}\left(U\left(\mathbf{C}_{p}\right)\right)\right)\right)$ has dimension strictly less than $\operatorname{deg}(\zeta)$.

If $V \subset \Gamma$ is an open edge, then the open annulus $U=\tau^{-1}(V)$ is a basic wide open subdomain with respect to any type- 2 point $\zeta \in V$. In this case one has the following slightly more precise variant of Proposition 3.28, recovering a result of Stoll [Sto13, Proposition 7.3]. 
Proposition 3.29. Let $e \subset \Gamma$ be an open edge, and let $A=\tau^{-1}(e) \subset X^{\text {an }}$, an open annulus. Choose an identification $A \cong \mathbf{S}(\varpi)_{+}$with the standard open annulus of inner radius $|\varpi|$ and outer radius 1 . Then for all $\omega \in \Omega_{X / \mathbf{C}_{p}}^{1}(X)$ there exists $a(\omega) \in \mathbf{C}_{p}$ such that

$$
\int_{x}^{y} \omega-\int_{x}^{\mathrm{BC}} \omega=a(\omega)(\operatorname{val}(y)-\operatorname{val}(x))
$$

for all $x, y \in \mathbf{S}(\varpi)_{+}\left(\mathbf{C}_{p}\right)$. Moreover, $\omega \mapsto a(\omega)$ is $\mathbf{C}_{p}$-linear.

The proof is almost identical to that of Proposition 3.28 and is left to the reader.

Remark 3.30. We expect that the above results should make it possible to compute abelian integrals on hyperelliptic curves of bad reduction in residue characteristic greater than 2. Such curves have an explicit cover by hyperelliptic wide opens that can be obtained from their defining equations (see [Sto13]). The Balakrishnan-Bradshaw-Kedlaya algorithm [BBK10] can be applied to such wide opens to compute Berkovich-Coleman integrals. After determining the tropical Abel-Jacobi map through the use of tropical 1-forms (see [MZ08]), one can then obtain the abelian integrals.

\section{Bounding ZERos of INTEGRALS ON WIDE OPENS}

In this section, we leverage Proposition 2.22 to bound the number of zeros of the BerkovichColeman integral of an exact 1-form $\omega=d f$ on a basic wide open curve. This amounts to relating the slopes of $-\log \|\omega\|$ to those of $-\log |f|$ on an annulus, which we do in Proposition 4.7. To eventually obtain bounds depending essentially only on the genus, we will also need a combinatorial argument about stable metric graphs, which we make in Lemmas 4.14 and 4.15. The main result of the section is Theorem 4.17.

In this section we work over $K=\mathbf{C}_{p}$.

4.1. Slopes on annuli. First, we recall the relationship between Newton polygons and slopes on the skeleton of an annulus. Let $\varpi \in \mathbf{C}_{p}^{\times}$with $|\varpi|<1$, and recall that $\mathbf{S}(\varpi)_{+}$ denotes the open annulus of outer radius 1 and inner radius $|\varpi|$. Let $a=\operatorname{val}(\varpi)$, the logarithmic modulus of $\mathbf{S}(\varpi)_{+}$. An analytic function on $\mathbf{S}(\varpi)_{+}$can be expressed as an infinite-tailed Laurent series $\sum_{n \in \mathbf{Z}} a_{n} T^{n}$ with the property that

$$
\operatorname{val}\left(a_{n}\right)+n r \rightarrow \infty \quad \text { as } \quad n \rightarrow \pm \infty
$$

for all $r \in(0, a)$. For $r \in(0, a)$, we set

$$
\left\|\sum a_{n} T^{n}\right\|_{r}=\max \left\{\left|a_{n}\right| \exp (-n r)\right\} .
$$

This is a multiplicative seminorm which defines a point $\xi_{r} \in \mathbf{S}(\varpi)_{+}$. The map $\sigma:(0, a) \rightarrow$ $\mathbf{S}(\varpi)_{+}$given by $\sigma(r)=\xi_{r}$ is a continuous embedding and its image $\Sigma\left(\mathbf{S}(\varpi)_{+}\right):=\sigma((0, a))$ is by definition the skeleton of $\mathbf{S}(\varpi)_{+}$.

Note that if $f(T)=\sum a_{n} T^{n}$ is an analytic function on $\mathbf{S}(\varpi)_{+}, F=-\log |f|$, and $\xi_{r}=$ $\sigma(r) \in \Sigma\left(\mathbf{S}(\varpi)_{+}\right)$, then by definition

$$
F\left(\xi_{r}\right)=-\log \|f\|_{r}=\min \left\{\operatorname{val}\left(a_{n}\right)+n r: a_{n} \neq 0\right\} .
$$


See Figure 2 for an illustration in terms of Newton polygons.

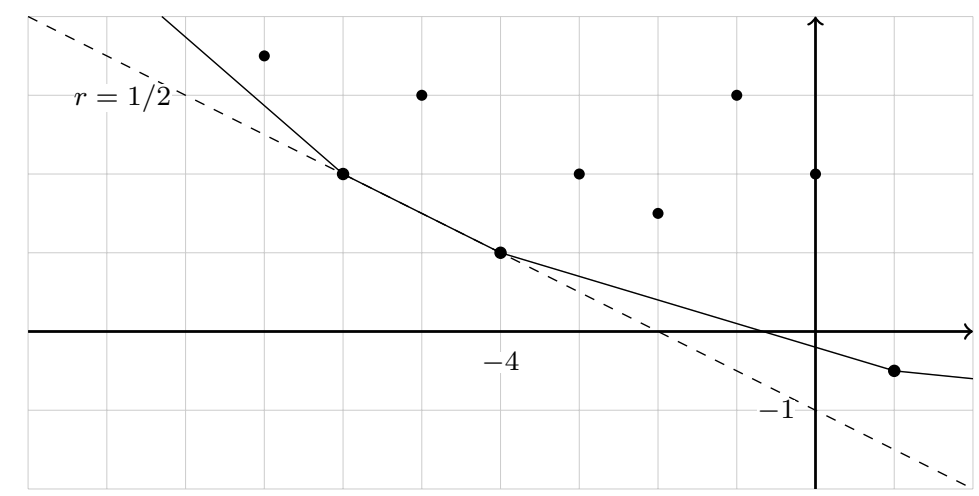

Figure 2. A possible Newton polygon of an analytic function $f=\sum a_{n} T^{n}$ on an open annulus. The dashed line is $y+\frac{1}{2} x=-1$. If $F=-\log |f|$, then $F\left(\xi_{1 / 2}\right)=-1$, and $d_{v} F\left(\xi_{1 / 2}\right)=4$ in the notation of Lemma 4.2 .

Recall that if $v$ is a tangent direction at $\xi_{r}$, then $d_{v} F\left(\xi_{r}\right)$ denotes the slope of $F$ in the $v$ direction.

Lemma 4.2. Let $\xi_{r}=\sigma(r) \in \Sigma\left(\mathbf{S}(\varpi)_{+}\right)$, and let $v$ be the tangent direction at $\xi_{r}$ defined by the line segment $\sigma((0, r])$. Let $f(T)=\sum a_{n} T^{n}$ be an analytic function on $\mathbf{S}(\varpi)_{+}$, and let $F=-\log |f|$. Then

$$
d_{v} F\left(\xi_{r}\right)=-\max \left\{n: \operatorname{val}\left(a_{n}\right)+n r=F\left(\xi_{r}\right)\right\} .
$$

Proof. Let $N=\max \left\{n: \operatorname{val}\left(a_{n}\right)+n r=F\left(\xi_{r}\right)\right\}$. There exists a small $\epsilon$ such that $\operatorname{val}\left(a_{N}\right)+$ $N s<\operatorname{val}\left(a_{n}\right)+n s$ for all $n \neq N$ and all $s \in(r-\epsilon, r)$. It follows that the restriction of $f$ to the subannulus

$$
A=\left\{\eta \in \mathbf{S}(\varpi)_{+}:-\log |T(\eta)| \in(r-\epsilon, r)\right\}
$$

is invertible, with $|f(\eta)|=\left|a_{N} \eta^{N}\right|$ for all $\eta \in A$. Therefore the slope of $-\log |f|$ along $\sigma((r-\epsilon, r))$ (in the positive direction) is equal to $N$ by [BPR13, Proposition 2.5(1)].

All of our bounds will be stated in terms of the following function $N_{p}\left(r, N_{0}\right)$.

Definition 4.3. Let $r$ be a positive real number, let $N_{0}$ be an integer, and let $p$ be a prime. Define $N_{p}\left(r, N_{0}\right)$ to be the smallest positive integer $N$ such that for all $n \geq N$, one has

$$
r\left(n-N_{0}\right)>\left\lfloor\log _{p}(n)\right\rfloor .
$$

Remark 4.5. The integer $N_{p}\left(r, N_{0}\right)$ gets larger as $N_{0}$ increases and as $r$ decreases, and it gets smaller as $p$ increases. Clearly

$$
\lim _{s \nearrow r} N_{p}\left(s, N_{0}\right)=N_{p}\left(r, N_{0}\right) .
$$

If $N_{0} \geq 0$ and $p \geq N_{0}+2$, then $N_{p}\left(r, N_{0}\right)=N_{0}+1$ because $\left\lfloor\log _{p}\left(N_{0}+1\right)\right\rfloor=0<r$. One should think of $N_{p}\left(r, N_{0}\right)-N_{0}$ as the correction to the $p$-adic Rolle theorem coming from the fact that $1 / p$ has negative valuation (see Corollary 4.13 and see also [Sto06, Section 6] 
for a more sophisticated approach to the same problem). (Stoll's correction factor $\delta(\cdot, \cdot)$ is slightly better, but ours is easier to define.)

We give an explicit upper bound on $N_{p}\left(r, N_{0}\right)$ as follows. If $N_{0} \leq 0$, we take $N=1$. Otherwise, write $N=N_{0} \exp (u)$ for $u>0$. We want $N-N_{0}>\frac{1}{r \ln (p)} \ln (N)$; that is,

$$
\exp (u)-1>\frac{\ln (N)}{N_{0} r \ln (p)}=\frac{\ln \left(N_{0}\right)}{N_{0} r \ln (p)}+\frac{u}{N_{0} r \ln (p)} .
$$

Writing $\exp (u)-1>u+\frac{u^{2}}{2}$ and using $u$ and $u^{2}$ to bound each term on the right, it suffices to pick

For $N_{0} \leq 7$, this gives

$$
u \geq \max \left(\frac{\ln \left(N_{0}\right)}{N_{0} r \ln (p)}, \frac{2}{N_{0} r \ln (p)}\right) .
$$

$$
N_{p}\left(r, N_{0}\right) \leq\left\lceil N_{0} \exp (u)\right\rceil=\left\lceil N_{0} \exp \left(\frac{2}{N_{0} r \ln (p)}\right)\right\rceil,
$$

while for $N_{0} \geq 8$, we have

$$
N_{p}\left(r, N_{0}\right) \leq\left\lceil N_{0} \exp (u)\right\rceil=\left\lceil N_{0}^{1+1 /\left(N_{0} r \ln (p)\right)}\right\rceil .
$$

If we suppose that $r \ln (p) \geq 1$, then one checks case by case that

$$
N_{p}\left(r, N_{0}\right) \leq 2 N_{0}
$$

for all $N_{0} \geq 1$.

In the statement of the next proposition we will use the following notation (see Figure 3):

$X$ A smooth, proper, connected $\mathbf{C}_{p}$-curve.

$\mathfrak{X}$ A semistable $\mathscr{O}_{\mathbf{C}_{p}}$-model of $X$.

$\Gamma=\Gamma_{\mathfrak{X}} \subset X^{\text {an }}$, a skeleton of $X$ in the sense of Section 2.2.

$\bar{e} \subset \Gamma$, a closed interval with type- 2 endpoints.

$\zeta_{ \pm}$The endpoints of $\bar{e}$.

$v_{ \pm}$The tangent direction at $\zeta_{ \pm}$in the direction of $e$.

$e=\bar{e} \backslash\left\{\zeta_{ \pm}\right\}$, the open interval inside $e$.

$A=\tau^{-1}(e) \cong \mathbf{S}(\varpi)_{+}$, an open annulus.

$a=\operatorname{val}(\varpi)$, the logarithmic modulus of $A$.

We choose an identification $A \cong \mathbf{S}(\varpi)_{+}$such that $\xi_{r} \rightarrow \zeta_{-}$as $r \rightarrow 0$, so $\xi_{r} \rightarrow \zeta_{+}$as $r \rightarrow a$. If $e$ is a loop edge, then $\zeta_{+}=\zeta_{-}$, and we define $v_{ \pm}$to be the two tangent directions at $\zeta_{ \pm}$in the direction of $e$. In what follows we use the formal metric $\|\cdot\|$ on $\Omega_{X / \mathbf{C}_{p}}^{1}$ induced by the sheaf of integral Rosenlicht differentials on $\mathfrak{X}$, as in Section 2.4 .

Proposition 4.7. With the above notation, let $\omega \in H^{0}\left(X, \Omega_{X / \mathbf{C}_{p}}^{1}\right)$ be a nonzero global differential, and suppose that $\omega$ is exact on $A$, so $\omega=d f$ for an analytic function $f$ on $A$. Let $F=-\log |f|$ and $F_{0}=-\log \|\omega\|$, and let $N_{0}=d_{v_{+}} F_{0}\left(\zeta_{+}\right)$. Choose $r \in(0, a)$, and let $v_{r}$ be the tangent direction at $\xi_{r}$ in the direction of $\zeta_{-}$. Then $d_{v_{r}} F\left(\xi_{r}\right) \leq N_{p}\left(a-r, N_{0}\right)$. 


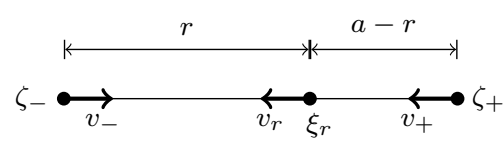

Figure 3. Illustration of the notation used in Proposition 4.7. The interval represents the edge $e$, which has length $a$.

Proof. Let $T: A \stackrel{\sim}{\longrightarrow} \mathbf{S}(\varpi)_{+}$be the identification we chose above, so $\xi \rightarrow \zeta_{-}$as $-\log |T(\xi)| \rightarrow$ 0 . The restriction of $\omega$ to $A$ has an infinite-tailed Laurent series expansion of the form

$$
\omega=\sum_{n \in \mathbf{Z}} a_{n} T^{n} \frac{d T}{T} .
$$

By Lemmas 2.14 and 2.15, for $\xi \in A$ one has $F_{0}(\xi)=-\log \|\omega(\xi)\|=-\log \left|\sum a_{n} \xi^{n}\right|$. Using (4.1) and taking the limit as $r \rightarrow a$, we obtain

$$
F_{0}\left(\zeta_{+}\right)=-\log \left\|\omega\left(\zeta_{+}\right)\right\|=\inf \left\{\operatorname{val}\left(a_{n}\right)+n a: a_{n} \neq 0\right\}
$$

In particular, the right-hand side of this equation is finite. Since $\omega$ has finitely many zeros on $A$, the Newton polygon $\mathcal{N}$ of $\sum a_{n} T^{n}$ has finitely many segments with slope in $(-a, 0)$. Therefore the infimum in (4.8) is achieved, and $d_{v_{+}} F_{0}\left(\zeta_{+}\right)=d_{v_{r^{\prime}}} F_{0}\left(\xi_{r^{\prime}}\right)$ for $r^{\prime}<a$ very close to $a$, where $\xi_{r^{\prime}}$ and $v_{r^{\prime}}$ are defined as in the statement of the proposition. From this and Lemma 4.2, as applied to $\sum a_{n} T^{n}$ and $\xi_{r^{\prime}}$ with $r^{\prime} \rightarrow a$, one sees that

$$
N_{0}=-\max \left\{n: \operatorname{val}\left(a_{n}\right)+n a=F_{0}\left(\zeta_{+}\right)\right\} .
$$

Since $d f=\omega$, we have

$$
f=\sum_{n \in \mathbf{Z}} b_{n} T^{n}=b_{0}+\sum_{n \neq 0} \frac{a_{n}}{n} T^{n}
$$

on $A$, where $b_{n}=a_{n} / n$ for $n \neq 0$ and $b_{0} \in \mathbf{C}_{p}$ is some constant. According to Lemma 4.2,

$$
d_{v_{r}} F\left(\xi_{r}\right)=-\max \left\{n: \operatorname{val}\left(b_{n}\right)+n r=F\left(\xi_{r}\right)\right\},
$$

where

$$
F\left(\xi_{r}\right)=\min \left\{\operatorname{val}\left(b_{n}\right)+n r: b_{n} \neq 0\right\} .
$$

The number $N:=N_{p}\left(a-r, N_{0}\right)$ is positive, so if $d_{v_{r}} F\left(\xi_{r}\right) \leq 0$, then we are done. Hence, we may assume $d_{v_{r}} F\left(\xi_{r}\right)>0$, so that val $\left(b_{n}\right)+n r=F\left(\xi_{r}\right)$ implies $n<0$. Note that we are in a situation where the constant $b_{0}$ plays no role. For $n<0$ such that $a_{n} \neq 0$, we have

$$
\begin{aligned}
\operatorname{val}\left(b_{n}\right)+n r & =\operatorname{val}\left(a_{n}\right)+n a-\operatorname{val}(n)-n(a-r) \\
& \geq \operatorname{val}\left(a_{-N_{0}}\right)-N_{0} a-\operatorname{val}(n)-n(a-r) \\
& =\operatorname{val}\left(a_{-N_{0}}\right)-N_{0} r-\operatorname{val}(n)-\left(n+N_{0}\right)(a-r) \\
& \geq \operatorname{val}\left(a_{-N_{0}}\right)-\operatorname{val}\left(-N_{0}\right)-N_{0} r-\operatorname{val}(n)-\left(n+N_{0}\right)(a-r) \\
& =\operatorname{val}\left(b_{-N_{0}}\right)-N_{0} r-\operatorname{val}(n)+\left(-n-N_{0}\right)(a-r) \\
& \geq F\left(\xi_{r}\right)-\left\lfloor\log _{p}(-n)\right\rfloor+\left(-n-N_{0}\right)(a-r) .
\end{aligned}
$$


Here we have used (4.8) and (4.9) in the first inequality, and (4.10) and (4.11) in the last (along with $\left.\operatorname{val}(n) \leq\left\lfloor\log _{p}(-n)\right\rfloor\right)$. It follows that when (4.4) is satisfied, then $\operatorname{val}\left(b_{n}\right)+n r>$ $F\left(\xi_{r}\right)$ for $n \leq-N$, so that $N \geq d_{v_{r}} F\left(\xi_{r}\right)$.

We would like to apply Proposition 4.7 to arbitrary open annuli embedded in $X^{\text {an }}$. For this we need the following lemma.

Lemma 4.12. Let $U \subset X^{\text {an }}$ be an open subdomain isomorphic to an open annulus $\mathbf{S}(\varpi)_{+}$. Then there exists a skeleton $\Gamma$ of $X$ and an open edge e of $\Gamma$ such that $\tau^{-1}(e)=U$.

Proof. First we recall that if $U^{\prime} \subset X^{\text {an }}$ is an open subdomain isomorphic to the open disc $\mathbf{B}(1)_{+}$, then the closure of $U^{\prime}$ is $U^{\prime} \amalg\{x\}$ for a type-2 point $x \in X^{\text {an }}$ by [ABBR15, Lemma 3.3].

By ABBR15, Lemma 3.6], the closure of $U$ in $X^{\text {an }}$ is $U \amalg\{x, y\}$, where $x, y \in X^{\text {an }}$ are points which are not necessarily distinct. We claim that $x, y$ have type 2 . This claim reduces to the case of a disc by doing surgery on $X^{\text {an }}$, as in the proof of [BPR13, Lemma 4.12(2)]. Briefly, one excises a closed subannulus from $U$, then caps the ends of the remaining two open annuli by open discs. One obtains a new open set $U^{\prime} \cong \mathbf{B}(1)_{+} \amalg \mathbf{B}(1)_{+}$in a new curve $X^{\prime a n}$, with $x, y$ identified with the points in the closures of the two open discs.

Let $V$ be a semistable vertex set of $X$ containing $x$ and $y$. Such exists by BPR13, Proposition 3.13(3)]. Let $V^{\prime}=V \backslash U$. Then $X^{\text {an }} \backslash V^{\prime}$ is again a disjoint union of open discs and open annuli, one of which is $U$, so $V^{\prime}$ is a semistable vertex set. The corresponding skeleton $\Gamma$ has an open edge $e:=\Gamma \cap U$ satisfying the conditions of the lemma.

As an immediate consequence we recover a general version of the standard ChabautyColeman bound for zeros of an antiderivative on an open disc, as found (in a slightly stronger version) in [Sto06, Proposition 6.3].

Corollary 4.13. Let $B \subset X^{\text {an }}$ be an open subset isomorphic to the open unit disc $\mathbf{B}(1)_{+}$, and choose an isomorphism $T: B \rightarrow \mathbf{B}(1)_{+}$. Let $\omega \in H^{0}\left(X, \Omega_{X / \mathbf{C}_{p}}^{1}\right)$ be a nonzero global differential, and let $N_{0}$ be the number of zeros of $\omega$ on $B$. Then $\omega=d f$ for an analytic function $f$ on $B$, and for any $r>0, f$ has at most $N_{p}\left(r, N_{0}+1\right)$ zeros on the subdisc $B_{r}:=\{\eta \in B:-\log |T(\eta)|>r\}$.

Proof. That $\omega$ is exact follows from the Poincaré lemma. Let $g$ be an analytic function on a disc $\mathbf{B}(1)_{+}$with finitely many zeros. By a classical Newton polygon argument, the number of zeros of $g$ on $\mathbf{B}(1)_{+}$is equal to the slope of $-\log |g|$ at the Gauss point $\zeta_{r}$ of the closed disc of radius $\exp (-r)$ for $r>0$ close to zero. Hence, the corollary follows from Proposition 4.7 as applied to an annulus of logarithmic modulus $a>r$ contained in $B$, recalling that the slope of $\omega$ on an annulus is calculated with respect to $d T / T$.

4.2. Combinatorics of stable graphs. The minimal skeleton $\Gamma=\Gamma_{\min }$ (in the sense of Section 2.2) of a curve of genus $g \geq 2$ is the skeleton associated to a stable model. This implies that $\Gamma$ is a connected metric graph, with vertices $x$ weighted by the genus $g(x)$, such that all vertices of valency $\leq 2$ have positive weight. Such a metric graph is called stable.

In this subsection we make some (undoubtedly well known) observations about the combinatorics of stable vertex-weighted metric graphs $(\Gamma, g)$. We extend the weight $g$ to all points 
of $\Gamma$ by setting $g(x)=0$ if $x$ is not a vertex. Likewise, we declare that the valency of a nonvertex $x \in \Gamma$ is $\operatorname{deg}(x):=2$. The genus of $\Gamma$ is defined via the genus formula (2.1): that is,

$$
g(\Gamma):=h_{1}(\Gamma)+\sum_{x \in \Gamma} g(x)
$$

Recall (2.17) that the canonical divisor on $\Gamma$ is

$$
K_{\Gamma}:=\sum_{x \in \Gamma}(2 g(x)-2+\operatorname{deg}(x))(x) .
$$

The degree of $K_{\Gamma}$ is $2 g(\Gamma)-2$, and since $\Gamma$ is stable, $K_{\Gamma}$ is effective and has positive multiplicity on every vertex.

Lemma 4.14. Let $(\Gamma, g)$ be a stable vertex-weighted metric graph of genus $g(\Gamma) \geq 2$.

(1) $\Gamma$ has at most $2 g-2$ vertices.

(2) $\Gamma$ has at most $3 g-3$ edges and at most $g$ loop edges.

(3) Every vertex of $\Gamma$ has valency at most $2 g(\Gamma)$.

Proof. As mentioned above, the canonical divisor $K_{\Gamma}$ has degree $2 g(\Gamma)-2$ and is effective, with positive multiplicity on vertices. Since $2 g(x)-2+\operatorname{deg}(x)=0$ for $x$ not a vertex, $K_{\Gamma}$ is supported on the set of vertices. This proves (1). Letting $V$ be the number of vertices of $\Gamma$ and $E$ be the number of edges, we have $h_{1}(\Gamma)=E-V+1$, so

$$
E=h_{1}(\Gamma)+V-1 \leq g(\Gamma)+(2 g(\Gamma)-2)-1=3 g(\Gamma)-3 .
$$

Clearly a graph with more than $g$ loop edges has genus greater than $g$, so this proves (2). For (3), note that

$$
2 g(\Gamma)-2=\operatorname{deg}\left(K_{\Gamma}\right)=\sum(2 g(x)-2+\operatorname{deg}(x)),
$$

where the sum is taken over all vertices. Since each summand is positive, for a given vertex $x$, we have $2 g(x)-2+\operatorname{deg}(x) \leq 2 g(\Gamma)-2$, so

$$
\operatorname{deg}(x) \leq 2 g(\Gamma)-2 g(x) \leq 2 g(\Gamma)
$$

The following lemma does not require the weighted metric graph to be stable. It plays the role of [Sto13, Corollary 6.7], which is proved using an explicit calculation on hyperelliptic curves.

Lemma 4.15. Let $(\Gamma, g)$ be a vertex-weighted metric graph of genus $g(\Gamma)$. Let $F$ be a tropical meromorphic function on $\Gamma$ such that $\operatorname{div}(F)+K_{\Gamma} \geq 0$. Then for all $x \in \Gamma$ and all tangent directions $v$ at $x$, we have $\left|d_{v} F(x)\right| \leq 2 g(\Gamma)-1$. If $K_{\Gamma}$ is effective, that is, if $\Gamma$ has no genus-zero leaves, then we may replace $2 g(\Gamma)-1$ by $2 g(\Gamma)-2$.

Proof. We may assume that $x$ is not a vertex and that $F$ is differentiable at $x$. First we assume that $\Gamma$ has no leaves of genus zero, so that $K_{\Gamma}$ is effective. If $F$ is constant in a neighborhood of $x$, then we are done, so assume that this is not the case. Let $r=F(x)$, 
let $\Gamma_{\leq r}=\{y \in \Gamma: F(y) \leq r\}$, and define $\Gamma_{<r}$ similarly. Then $\Gamma_{\leq r}$ is a subgraph of $\Gamma, x$ is a leaf of $\Gamma_{\leq r}$, and the tangent direction $v$ at $x$ in which $F$ is increasing points away from $\Gamma_{\leq r}$. Let $x_{1}, \ldots, x_{n}$ be the points on the boundary of $\Gamma_{\leq r}$ in $\Gamma$, and let $\left\{v_{i j}\right\}$ be the tangent directions at $x_{i}$ in $\Gamma_{\leq r}$. The degree of the tropical meromorphic function $\left.F\right|_{\Gamma_{\leq r}}$ on the metric graph $\Gamma_{\leq r}$ is zero, so we have

$$
0=\sum_{y \in \Gamma_{\leq r}} \operatorname{ord}_{y}(F)=-\sum d_{v_{i j}} F\left(x_{i}\right)+\sum_{y \in \Gamma<r} \operatorname{ord}_{y}(F),
$$

since $\operatorname{ord}_{v_{i j}}(F)$ is the incoming slope. As each $-d_{v_{i j}} F\left(x_{i}\right)$ is nonnegative, we have

$$
d_{v} F(x) \leq-\sum d_{v_{i j}} F\left(x_{i}\right)=-\sum_{y \in \Gamma_{<r}} \operatorname{ord}_{y}(F)
$$

Let $m_{y}=2 g(y)-2+\operatorname{deg}(y)$, the multiplicity of $y$ in $K_{\Gamma}$. Then $\operatorname{ord}_{y}(F)+m_{y} \geq 0$, so $-\operatorname{ord}_{y}(F) \leq m_{y}$ and hence,

$$
-\sum_{y \in \Gamma_{<r}} \operatorname{ord}_{y}(F) \leq \sum_{y \in \Gamma<r} m_{y} \leq 2 g(\Gamma)-2
$$

since $K_{\Gamma}$ has degree $2 g(\Gamma)-2$, and $m_{y} \geq 0$ for $y \notin \Gamma_{<r}$.

Now we drop the assumption that $\Gamma$ has no genus-zero leaves. Let $z$ be such a leaf, let $y$ be the first vertex along the edge adjoining $z$, that is, the first point along this edge with $m_{y} \neq 0$, and let $e$ be the line segment joining $y$ and $z$. The lemma is easy to prove when $\Gamma=e$, so we assume this is not the case. Since $m_{z}=-1$, the incoming slope of $F$ at $z$ is at least 1. From this it follows that $F$ is monotonically increasing from $y$ to $z$, and in particular, that the incoming slope of $F$ at $y$ is at most -1 . Letting $\Gamma^{\prime}=(\Gamma \backslash e) \cup\{y\}$, this implies that $\left.F\right|_{\Gamma^{\prime}}$ is a tropical meromorphic function satisfying $\operatorname{div}\left(\left.F\right|_{\Gamma^{\prime}}\right)+K_{\Gamma^{\prime}} \geq 0$.

Let $x \in \Gamma$. By repeatedly removing genus-zero leaf edges not containing $x$, we may find a subgraph $\Gamma^{\prime \prime} \subseteq \Gamma$ containing $x$ with at most one genus-zero leaf edge $e$ (which then contains $x$ by construction) such that $\left.F\right|_{\Gamma^{\prime \prime}}$ is a tropical meromorphic function satisfying $\operatorname{div}\left(\left.F\right|_{\Gamma^{\prime \prime}}\right)+K_{\Gamma^{\prime \prime}} \geq 0$. Note that $g\left(\Gamma^{\prime \prime}\right)=g(\Gamma)$ and that $\Gamma^{\prime}$ has at most one point $z$ with $m_{z}<0$. If there is no such point, then the conclusion follows from the special case above. Otherwise, we proceed as before. Because $m_{z}=-1$, we obtain

$$
-\sum_{y \in\left(\Gamma^{\prime \prime}\right)_{<r}} \operatorname{ord}_{y}(F) \leq \sum_{y \in\left(\Gamma^{\prime \prime}\right)_{<r}} m_{y} \leq 2 g\left(\Gamma^{\prime \prime}\right)-2+1=2 g(\Gamma)-1 .
$$

4.3. Bounding zeros on wide opens. Let $U$ be a basic wide open subdomain of $X^{\text {an }}$ with central point $\zeta$, underlying affinoid $Y$, and annuli $A_{1}, \ldots, A_{d}$, as in Section 2.5. Suppose that $U$ is defined with respect to a star neighborhood in a skeleton $\Gamma$.

Definition 4.16. The thickness of $U$ is $\min \left\{a_{1}, \ldots, a_{d}\right\}$, where $a_{i}$ is the logarithmic modulus of $A_{i}$. 
Let $a$ be the thickness of $U$. For $r \in(0, a) \cap \mathbf{Q}$, we let $U_{r}$ denote the basic wide open subdomain inside of $U$ obtained by deleting a half-open annulus of logarithmic modulus $r$ from each $A_{i}$, as in Figure 4 ,

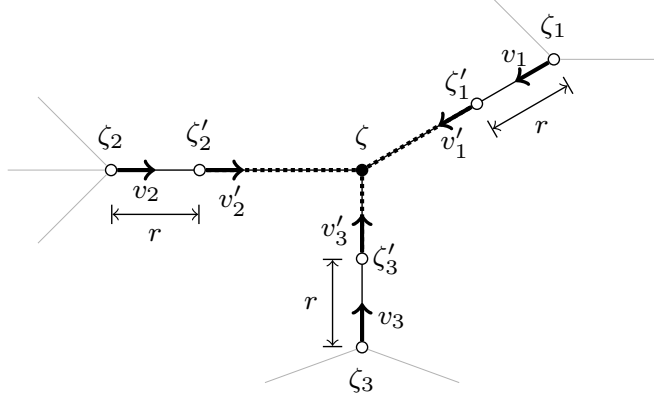

FiguRE 4. Illustration of Definition 4.16 and the proof of Theorem 4.17. The lines of standard thickness represent an open star neighborhood $V$ of $\zeta$ such that $U=\tau^{-1}(V)$. The dotted lines represent the smaller open star neighborhood $V_{r}$ of $\zeta$ such that $U_{r}=\tau^{-1}\left(V_{r}\right)$. The ends of $U$ (resp., $\left.U_{r}\right)$ are $\zeta_{1}, \zeta_{2}, \zeta_{3}$ $\left(\right.$ resp., $\left.\zeta_{1}^{\prime}, \zeta_{2}^{\prime}, \zeta_{3}^{\prime}\right)$.

Theorem 4.17. With the above notation, let $\omega \in H^{0}\left(X, \Omega_{X / \mathbf{C}_{p}}^{1}\right)$ be a nonzero global differential, and suppose that $\omega$ is exact on $U$, so $\omega=d f$ for an analytic function $f$ on $U$. Then $f$ has at most $\operatorname{deg}(\zeta) N_{p}(r, 2 g-1)$ geometric zeros, counted with multiplicity, on $U_{r}$, where $\operatorname{deg}(\zeta)$ is the valency of $\zeta$ in $\Gamma$. If $U$ is defined with respect to a star neighborhood in a skeleton with no genus-zero leaves, then we may replace $2 g-1$ by $2 g-2$.

Proof. Let $F_{0}=-\log \|\omega\|$, and let $F=-\log |f|$, as in the statement of Proposition 4.7. As explained in Section 2.4.2, $F_{0}$ is a section of the tropical canonical bundle on $\Gamma$; that is, it satisfies the hypotheses of Lemma 4.15. Hence, the absolute value of the slope of $F_{0}$ in any direction at any point of $\Gamma$ is at most $2 g-1$, or $2 g-2$ if $\Gamma$ has no genus-zero leaves. In the latter case one may replace $2 g-1$ by $2 g-2$ everywhere below.

Let $d=\operatorname{deg}(\zeta)$, let $\zeta_{1}, \ldots, \zeta_{d}$ (resp., $\left.\zeta_{1}^{\prime}, \ldots, \zeta_{d}^{\prime}\right)$ be the ends of $U$ (resp., $U_{r}$ ), and let $v_{i}$ (resp., $v_{i}^{\prime}$ ) be the tangent direction at $\zeta_{i}$ (resp., $\zeta_{i}^{\prime}$ ) pointing in the direction of the central point $\zeta$, as in Figure 4, By the above, we have $d_{v_{i}} F\left(\zeta_{i}\right) \leq 2 g-1$ for all $i=1, \ldots, d$. By Proposition 4.7 ,

$$
d_{v_{i}^{\prime}} F\left(\zeta_{i}^{\prime}\right) \leq N_{p}\left(r, d_{v_{i}} F\left(\zeta_{i}\right)\right) \leq N_{p}(r, 2 g-1)
$$

for all $i=1, \ldots, d$. By Proposition 2.22, then,

$$
\operatorname{deg}\left(\operatorname{div}\left(\left.f\right|_{U_{r}}\right)\right)=\sum_{i=1}^{d} d_{v_{i}^{\prime}} F\left(\zeta_{i}^{\prime}\right) \leq d N_{p}(r, 2 g-1) .
$$

The following corollary plays the role of [Sto13, Proposition 7.7], with the slope bound of Lemma 4.15 replacing [Sto13, Corollary 6.7]. 
Corollary 4.18. In the setting of Theorem 4.17, if $U$ is an open annulus, then $f$ has at most $2 N_{p}(r, 2 g-1)$ zeros on $U_{r}$.

By Lemma 4.12, any open subdomain $U \subset X^{\text {an }}$ which is isomorphic to an open annulus has the form $\tau^{-1}(e)$ for an open edge $e$ of some skeleton $\Gamma$ of $X$. This is a basic wide open subdomain with respect to the star neighborhood $e$ of the midpoint $\zeta$ of $e$. Therefore Corollary 4.18 applies to any embedded open annulus.

\section{UNIFORM BOUNDS}

In this section we use the following notation:

$K \quad$ A local field of characteristic 0 .

$\varpi$ A uniformizer of $K$.

$k$ The residue field of $K$.

$p$ The characteristic of $k$.

$q$ The number of elements of $k$.

$e$ The ramification degree of $\mathscr{O}_{K}$ over $\mathbf{Z}_{p}$.

$X$ A smooth, proper, geometrically connected curve over $K$.

$g$ The genus of $X$, assumed to be $\geq 2$.

$J$ The Jacobian of $X$.

$\iota: X \hookrightarrow J$, an Abel-Jacobi map defined over $K$.

We normalize the valuation on $K$ such that $\operatorname{val}(p)=1$, and we fix an isometric embedding $K \hookrightarrow \mathbf{C}_{p}$. Recall that $N_{p}(\cdot, \cdot)$ is defined in Definition 4.3.

5.1. Uniform bounds on $K$-rational points. In the following theorem we combine Corollary 4.18 and [Sto13, Proposition 5.3] to obtain uniform bounds on the number of $K$-points of $X$ mapping into a subgroup of $J(K)$ of a given rank $\rho$. This generalizes [Sto13, Theorem 9.1].

Theorem 5.1. Let $G \subset J(K)$ be a subgroup of rank $\rho \leq g-3$. Then

$$
\# \iota^{-1}(G) \leq(5 q g+6 g-2 q-8) N_{p}(1 / e, 2 g-1) .
$$

Proof. We will use $X^{\text {an }}$ to denote the $\mathbf{C}_{p}$-analytic space $\left(X \otimes_{K} \mathbf{C}_{p}\right)$ an. Let $V$ be the annihilator in $H^{0}\left(X_{\mathbf{C}_{p}}, \Omega_{X_{\mathbf{C}_{p}} / \mathbf{C}_{p}}^{1}\right)=H^{0}\left(J_{\mathbf{C}_{p}}, \Omega_{J_{\mathbf{C}_{p}} / \mathbf{C}_{p}}^{1}\right)$ of $\log _{J\left(\mathbf{C}_{p}\right)}(G)$, with the notation in Section 3.3. By the standard Chabauty-Coleman calculation, $V$ has dimension at least $g-\rho \geq 3$. Moreover, for $\omega \in V$ we have ${ }^{\mathrm{Ab}} \int_{x}^{y} \omega=0$ for all $x, y \in \iota^{-1}(G)$.

Let $B \subset X^{\text {an }}$ be an open subdomain defined over $K$ which is $K$-isomorphic to the open unit disc $\mathbf{B}(1)_{+}$. Suppose that there exists $x \in X(K) \cap B$. There is an isomorphism $T: B \stackrel{\sim}{\longrightarrow} \mathbf{B}(1)_{+}$such that $x \mapsto 0$ and $B \cap X(K)$ is identified with $\varpi \mathscr{O}_{K}$. In particular, $B \cap X(K) \subset B_{r}:=\{\eta \in B:-\log |T(\eta)|>r\}$ for all $r<\frac{1}{e}$. For any nonzero $\omega \in V$, there is a unique analytic function $f$ on $B$ such that $d f=\omega$ and $f(x)=0$. For $y \in B\left(\mathbf{C}_{p}\right)$, we have ${ }^{\mathrm{Ab}} \int_{x}^{y} \omega={ }^{\mathrm{BC}} \int_{x}^{y} \omega=f(y)$. Hence points of $\iota^{-1}(G)$ in $B$ are zeros of $f$, so

$$
\# \iota^{-1}(G) \cap B \leq \lim _{r \nearrow 1 / e} N_{p}(r,(2 g-2)+1)=N_{p}(1 / e, 2 g-1)
$$


by Corollary 4.13 ,

Now let $A \subset X^{\text {an }}$ be an open subdomain defined over $K$ which is $K$-isomorphic to an open annulus $\mathbf{S}\left(\varpi^{b}\right)_{+}$for $b \geq 1$. Then $A \cap X(K) \subset A_{r}:=\{\eta \in A:-\log |T(\eta)| \in(r, b / e-r)\}$ for all $r<\frac{1}{e}$ as above. Suppose that there exists $x \in A \cap X(K)$. This implies that $b \geq 2$. Choose $\omega \in V$ nonzero which is exact on $A$ and such that ${ }^{\mathrm{BC}} \int_{\gamma} \omega={ }^{\mathrm{Ab}} \int_{\gamma} \omega$ for all paths $\gamma$. This is possible because both are codimension-one conditions on $\omega$ : namely, that $\operatorname{Res}(\omega)=0$ in the notation of Section 2.6 and that $a(\omega)=0$ in the notation of Proposition 3.29, As above, there is an analytic function $f$ on $A$ such that $d f=\omega$ and all points of $\iota^{-1}(G)$ in $A$ are zeros of $f$. By Corollary 4.18, then,

$$
\# \iota^{-1}(G) \cap A \leq \lim _{r \nearrow 1 / e} 2 N_{p}(r, 2 g-1)=2 N_{p}(1 / e, 2 g-1) .
$$

By [Sto13, Proposition 5.3], there exists $t \in\{0,1,2, \ldots, g\}$ such that $X(K)$ is covered by at most $(5 q+2)(g-1)-3 q(t-1)$ embedded open discs and at most embedded $2 g-3+t$ open annuli, all defined over $K$. Using (5.2) and (5.3), then, we have

$$
\begin{aligned}
\# \iota^{-1}(G) & \leq((5 q+2)(g-1)-3 q(t-1)) N_{p}(1 / e, 2 g-1)+2(2 g-3+t) N_{p}(1 / e, 2 g-1) \\
& \leq((5 q+2)(g-1)-3 q(t-1)+4 g-6+2 t)) N_{p}(1 / e, 2 g-1) \\
& \leq(5 q g+6 g-2 q-8) N_{p}(1 / e, 2 g-1),
\end{aligned}
$$

where the third inequality holds because the quantity is maximized at $t=0$.

Suppose now that $X$ is defined over a number field $F$. Let $\mathfrak{p}$ be a prime of $F$ over 2 , and let $K=F_{\mathfrak{p}}$. The number $q$ of elements of the residue field $k$ of $F_{\mathfrak{p}}$ and the ramification degree of $F_{\mathfrak{p}}$ over $\mathbf{Z}_{2}$ are both bounded in terms of the degree $[K: \mathbf{Q}]$. Applying Theorem 5.1 with $G=J(F)$ yields Theorem [1.1, and applying Theorem 5.1 with $G=J(F)_{\text {tors }}$ yields Theorem 1.2 .

Remark 5.4. It should be possible to refine the bound of Theorem 5.1 to include the rank $\rho$, as in [Sto13, Theorem 8.1], although it is not obvious how to generalize Corollary 4.18 in this way.

5.2. Uniform bounds on geometric torsion packets. In the following theorem, the Abel-Jacobi map $\iota: X \hookrightarrow J$ need only be defined over $\mathbf{C}_{p}$. The requirement that $X$ be defined over $K$ and not just over $\mathbf{C}_{p}$ is only used to bound from below the minimum length of an edge in a skeleton $\Gamma$; the resulting bounds depend on $K$ only through its ramification degree over $\mathbf{Z}_{p}$. We set

$$
E(g, p):= \begin{cases}\# \mathrm{GSp}_{2 g}\left(\mathbf{F}_{5}\right) & \text { if } p \neq 5 \\ \# \mathrm{GSp}_{2 g}\left(\mathbf{F}_{7}\right) & \text { if } p=5 .\end{cases}
$$

Note that

$$
\# \mathrm{GSp}_{2 g}\left(\mathbf{F}_{\ell}\right)=\left(\ell^{2 g}-1\right)\left(\ell^{2 g-2}-1\right) \cdots\left(\ell^{2}-1\right) \cdot \ell^{g^{2}} \cdot(\ell-1)<\ell^{2 g^{2}+g+1}
$$

for any prime $\ell$. 
Theorem 5.5. Let $\Gamma$ be the minimal skeleton of $X_{\mathbf{C}_{p}}$, considered as a vertex-weighted metric graph.

(1) If $g>2 g(v)+\operatorname{deg}(v)$ for all vertices $v$ of $\Gamma$, then

$$
\# \iota^{-1}\left(J\left(\mathbf{C}_{p}\right)_{\text {tors }}\right) \leq\left(16 g^{2}-12 g\right) N_{p}\left((4 e E(g, p))^{-1}, 2 g-2\right) .
$$

(2) If $g>2 g(v)+2 \operatorname{deg}(v)-2$ for all vertices $v$ of $\Gamma$, then

$$
\# \iota^{-1}\left(J\left(\mathbf{C}_{p}\right)_{\text {tors }}\right) \leq(8 g-6) N_{p}\left((4 e E(g, p))^{-1}, 2 g-2\right) .
$$

Note that the bounds only depend on $p$ through the correction factor $N_{p}(\cdot, \cdot)$, which can be removed by recalling that $N_{2}(\cdot, \cdot) \geq N_{p}(\cdot, \cdot)$.

Proof. First suppose that $X$ admits a split stable model $\mathfrak{X}$ over $\mathscr{O}_{K}$, so that $\Gamma=\Gamma_{\mathfrak{X}}$. The hypotheses imply that $X$ does not have good reduction, namely, that $\Gamma$ is not a point. Let $\Gamma^{\prime}$ denote the metric graph obtained from $\Gamma$ by adding a vertex at the midpoint of each loop edge. Since $\Gamma$ is stable, $2 g(v)+\operatorname{deg}(v) \geq 3$ for all vertices $v$ of $\Gamma$, and $2 g(v)+\operatorname{deg}(v)=2$ if $v$ is a midpoint of a loop edge, so $g>2 g(v)+\operatorname{deg}(v)$ for all vertices of $\Gamma^{\prime}$. Note that $\Gamma^{\prime}$ has at most $3 g-2$ vertices and $4 g-3$ edges by Lemma 4.14. Since our model $\mathfrak{X}$ is split, each edge of $\Gamma^{\prime}$ has length at least $1 / 2 e$.

For each vertex $v$ of $\Gamma^{\prime}$, let $S_{v}$ denote the union of $v$ and all open edges adjacent to $v$, and let $U_{v}=\tau^{-1}\left(S_{v}\right)$. Then $U_{v}$ is a basic wide open subdomain of $X^{\text {an }}$ of thickness (Definition 4.16) at least $1 / 2 e$. By Theorem 2.24 the space $V_{v} \subset H^{0}\left(X_{\mathbf{C}_{p}}, \Omega_{X_{\mathbf{C}_{p}} / \mathbf{C}_{p}}^{1}\right)$ of 1 -forms $\omega$ which are exact on $U_{v}$ has dimension at least

$$
\operatorname{dim}\left(V_{v}\right) \geq g-(2 g(v)-1+\operatorname{deg}(v)) \geq 2 .
$$

Let $\epsilon$ be an open edge of $\Gamma^{\prime}$ adjacent to $v$, and let $U_{v, \epsilon}=\tau^{-1}(\{v\} \cup \epsilon) \subset U_{v}$, the union of the underlying affinoid of $U_{v}$ with the open annulus $\tau^{-1}(\epsilon)$. By Proposition 3.29, there exists a nonzero differential $\omega \in V_{v}$ such that ${ }^{\mathrm{BC}} \int_{x}^{y} \omega={ }^{\mathrm{Ab}} \int_{x}^{y} \omega$ for all $x, y \in U_{v, \epsilon}\left(\mathbf{C}_{p}\right)$.

Suppose that there exists $x_{0} \in U_{v, \epsilon}\left(\mathbf{C}_{p}\right)$ such that $\iota\left(x_{0}\right) \in J\left(\mathbf{C}_{p}\right)_{\text {tors }}$. Since $\omega$ is exact, we have $\omega=d f$ for an analytic function $f$ on $U_{v}$ such that $f\left(x_{0}\right)=0$. Since

$$
f(y)=\int_{x_{0}}^{\mathrm{BC}} \omega=\int_{x_{0}}^{\mathrm{Ab}} \omega=\left\langle\log _{J\left(\mathbf{C}_{p}\right)}\left(\iota(y)-\iota\left(x_{0}\right)\right), \omega\right\rangle
$$

for $y \in U_{v, \epsilon}\left(\mathbf{C}_{p}\right)$ and since $\log _{J\left(\mathbf{C}_{p}\right)}$ vanishes on $J\left(\mathbf{C}_{p}\right)_{\text {tors }}$, we have $f(y)=0$ for all $y \in$ $\iota^{-1}\left(J\left(\mathbf{C}_{p}\right)_{\text {tors }}\right) \cap U_{v, \epsilon}$. Choose $r \in(0,1 / 4 e)$, define $U_{v, r} \subset U_{v}$ as in Section 4.3, and let $U_{v, \epsilon, r}=U_{v, r} \cap U_{v, \epsilon}$. Then

$$
\#\left(\iota^{-1}\left(J\left(\mathbf{C}_{p}\right)_{\text {tors }}\right) \cap U_{v, \epsilon, r}\right) \leq \operatorname{deg}\left(\operatorname{div}\left(\left.f\right|_{U_{v, \epsilon, r}}\right)\right) \leq \operatorname{deg}\left(\operatorname{div}\left(\left.f\right|_{U_{v, r}}\right)\right) \leq 2 g N_{p}(r, 2 g-2),
$$

where we have used Theorem 4.17 and Lemma 4.14(3) for the final inequality. We have $X^{\text {an }}=\bigcup_{v, \epsilon} U_{v, \epsilon, r}$, where the union is taken over all vertices $v$ of $\Gamma^{\prime}$ and all open edges $\epsilon$ adjacent to $v$, and where $r \in(0,1 / 4 e)$ (recall that $1 / 4 e$ is half the minimum length of an edge). The number of pairs $(v, \epsilon)$ consisting of a vertex and an adjacent edge is equal to twice the number of edges, which is at most $8 g-6$. Therefore,

$$
\# \iota^{-1}\left(J\left(\mathbf{C}_{p}\right)_{\text {tors }}\right) \leq \underset{37}{(8 g-6)}(2 g) N_{p}(r, 2 g-2)
$$


for all $r<1 / 4 e$. Taking the limit as $r \nearrow 1 / 4 e$ yields assertion (1) in this case.

Now suppose that $g>2 g(v)+2 \operatorname{deg}(v)-2$ for all vertices $v$ of $\Gamma$ (hence of $\Gamma^{\prime}$ ). Then $\operatorname{dim}\left(V_{v}\right) \geq \operatorname{deg}(v)$, so by Proposition 3.28, there exists a nonzero differential $\omega \in V_{v}$ such that ${ }^{\mathrm{BC}} \int_{x}^{y} \omega={ }^{\mathrm{Ab}} \int_{x}^{y} \omega$ for all $x, y \in U_{v}\left(\mathbf{C}_{p}\right)$. Proceeding as above, we see that

$$
\#\left(\iota^{-1}\left(J\left(\mathbf{C}_{p}\right)_{\text {tors }}\right) \cap U_{v, r}\right) \leq \operatorname{deg}(v) N_{p}(r, 2 g-2)
$$

for all $r \in(0,1 / 4 e)$. Using the facts that $X^{\text {an }}=\bigcup_{v} U_{v, r}$ and that $\sum_{v} \operatorname{deg}(v)$ is twice the number of edges in $\Gamma^{\prime}$, we have

$$
\# \iota^{-1}\left(J\left(\mathbf{C}_{p}\right)_{\text {tors }}\right) \leq \sum_{v} \operatorname{deg}(v) N_{p}(r, 2 g-2) \leq(8 g-6) N_{p}(r, 2 g-2) .
$$

Taking the limit as $r \nearrow 1 / 4 e$ completes the proof in this case.

Finally, we reduce to the case when $X$ admits a split stable model over $K$ by making a potentially ramified field extension $K^{\prime} / K$. By [DM69, Theorem 2.4], $X$ admits a stable model over $\mathscr{O}_{K}$ if and only if its Jacobian $J$ has stable reduction, that is, if and only if the connected component of the special fiber of the Néron model of $J_{K}$ is semiabelian. By [SZ95, Corollary 6.3], for any prime $\ell \geq 5$ which is coprime to $p$, if $K^{\prime \prime}=K(J[\ell])$, then $J_{K^{\prime \prime}}$ admits a stable model. Since $J$ is principally polarized, $\operatorname{Gal}\left(K^{\prime \prime} / K\right) \subset \operatorname{GSp}_{2 g}\left(\mathbf{F}_{\ell}\right)$. Choosing $\ell=5$ or, if $p=5, \ell=7$, gives $\left[K^{\prime \prime}: K\right] \leq E(g, p)$. In particular, the ramification degree of $K^{\prime \prime} / K$ is at most $E(g, p)$, so the ramification degree of $K^{\prime \prime} / \mathbf{Z}_{p}$ is at most $e E(g, p)$. The stable model of $X_{K^{\prime \prime}}$ may not be split, but it can be made split by trivializing the action of $\mathrm{Gal}(\bar{k} / k)$ on the geometric skeleton $\Gamma$. This results in an unramified extension $K^{\prime}$ of $K^{\prime \prime}$. Now we apply the above argument to the curve $X_{K^{\prime}}$.

Remark 5.7. The hypotheses of Theorem 5.5(1) are satisfied if $X$ is a Mumford curve of genus $g$ and all vertices of $\Gamma$ have valency at most $g-1$, namely, if $g \geq 4$ and $\Gamma$ is trivalent. The hypotheses of Theorem 5.5)(2) are satisfied if $X$ is a Mumford curve of genus $g$ and all vertices of $\Gamma$ have valency at most $g / 2$, namely, if $g \geq 6$ and $\Gamma$ is trivalent.

Acknowledgements. This work clearly owes a debt to Stoll [Sto13, who, in addition to being an inspiration, had many helpful comments on an early draft of this paper. The authors would also like to thank Matt Baker, Vladimir Berkovich, Kiran Kedlaya, Dino Lorenzini, Andrew Obus, Jennifer Park, Bjorn Poonen, Alice Silverberg, and Yuri Zarhin for helpful discussions; Matt Baker and Walter Gubler for further comments on an early draft; and Christian Vilsmeier for important corrections. The authors thank the referees for a number of comments and corrections. Katz was supported by a National Sciences and Energy Research Council Discovery grant. Rabinoff was sponsored by a National Security Agency Young Investigator grant. Zureick-Brown was supported by a National Security Agency Young Investigator grant.

\section{REFERENCES}

[ABBR15] Omid Amini, Matthew Baker, Erwan Brugallé, and Joseph Rabinoff, Lifting harmonic morphisms I: metrized complexes and Berkovich skeleta, Res. Math. Sci. 2 (2015), Art. 7, 67. MR 3375652 2.4 .24 .1 
[Bak08] Matthew Baker, Specialization of linear systems from curves to graphs, Algebra Number Theory 2 (2008), no. 6, 613-653, With an appendix by Brian Conrad. 2.3, 2.4.3.

[BBK10] Jennifer S. Balakrishnan, Robert W. Bradshaw, and Kiran S. Kedlaya, Explicit Coleman integration for hyperelliptic curves, Algorithmic number theory, Lecture Notes in Comput. Sci., vol. 6197, Springer, Berlin, 2010, pp. 16-31.3.30

[Ber90] Vladimir G. Berkovich, Spectral theory and analytic geometry over non-Archimedean fields, Mathematical Surveys and Monographs, vol. 33, American Mathematical Society, Providence, RI, 1990. 1.1, 2.1, 2.2, 3.4.1, 3.4.2

[Ber07] _ Integration of one-forms on p-adic analytic spaces, Annals of Mathematics Studies, vol. 162, Princeton University Press, Princeton, NJ, 2007. 3.2

[BF11] Matthew Baker and Xander Faber, Metric properties of the tropical Abel-Jacobi map, J. Algebraic Combin. 33 (2011), no. 3, 349-381. 3.5.1, 3.5.1

[BG13] Manjul Bhargava and Benedict H. Gross, The average size of the 2-Selmer group of Jacobians of hyperelliptic curves having a rational Weierstrass point, Automorphic representations and $L$ functions, Tata Inst. Fundam. Res. Stud. Math., vol. 22, Tata Inst. Fund. Res., Mumbai, 2013, pp. 23-91. MR 3156850 1.5

[BGW13] Manjul Bhargava, Benedict H Gross, and Xiaoheng Wang, Pencils of quadrics and the arithmetic of hyperelliptic curves, preprint arXiv:1310.7692. To appear in J. Amer. Math. Soc. (2013). 1.5

[Bha13] Manjul Bhargava, Most hyperelliptic curves over $\mathbf{Q}$ have no rational points, preprint arXiv:1308.0395 (2013). 1.5

[BL84] Siegfried Bosch and Werner Lütkebohmert, Stable reduction and uniformization of abelian varieties. II, Invent. Math. 78 (1984), no. 2, 257-297. 3.4.1

[BL91] — Degenerating abelian varieties, Topology 30 (1991), no. 4, 653-698. 3.4.1, 3.4.2

[BL93] - Formal and rigid geometry. I. Rigid spaces, Math. Ann. 295 (1993), no. 2, 291-317.2.3

[BPR13] Matthew Baker, Sam Payne, and Joseph Rabinoff, On the structure of nonarchimedean analytic curves, Tropical and Non-Archimedean Geometry, Contemp. Math., vol. 605, Amer. Math. Soc., Providence, RI, 2013, pp. 93-121. 1.2, 2.2, 2.3, 2.3, 2.4.1, 2.4.1, 3.4.2, 4.1, 4.1

[BR10] Matthew Baker and Robert Rumely, Potential theory and dynamics on the Berkovich projective line, Mathematical Surveys and Monographs, vol. 159, American Mathematical Society, Providence, RI, 2010. 2.5

[BR14] Matthew Baker and Joseph Rabinoff, The skeleton of the Jacobian, the Jacobian of the skeleton, and lifting meromorphic functions from tropical to algebraic curves, Int. Math. Res. Not. IMRN (2014). 3.4.1, 3.5.1, 3.24, 3.5.1, 3.25

[Bre00] Christophe Breuil, Intégration sur les variétés p-adiques (d'après Coleman, Colmez), Astérisque (2000), no. 266, Exp. No. 860, 5, 319-350, Séminaire Bourbaki, Vol. 1998/99. 3.3

[BS13] Manjul Bhargava and Arul Shankar, The average size of the 5-Selmer group of elliptic curves is 6, and the average rank is less than 1, preprint arXiv:1312.7859 (2013). 1.5

[Bui96] Alexandru Buium, Geometry of p-jets, Duke Math. J. 82 (1996), no. 2, 349-367. 1, 1

[BZ] Amnon Besser and Sarah Zerbes, Vologodsky integration on semi-stable curves, in preparation. 3

[CdS88] Robert Coleman and Ehud de Shalit, $p$-adic regulators on curves and special values of p-adic L-functions, Invent. Math. 93 (1988), no. 2, 239-266. 3.2

[Cha41] Claude Chabauty, Sur les points rationnels des courbes algébriques de genre supérieur à l'unité, C. R. Acad. Sci. Paris 212 (1941), 882-885. 1.1

[CHM97] Lucia Caporaso, Joe Harris, and Barry Mazur, Uniformity of rational points, J. Amer. Math. Soc. 10 (1997), no. 1, 1-35. 1

[Chr13] Christian Christensen, Erste Chernform und Chambert-Loir Maße auf dem Quadrat einer TateKurve., Ph.D. thesis, Universität Tübingen, 2013, available at tobias-lib.uni-tuebingen.de. 2.3 
[CI99] Robert Coleman and Adrian Iovita, The Frobenius and monodromy operators for curves and abelian varieties, Duke Math. J. 97 (1999), no. 1, 171-215. 3.3 , 3.4

[CL06] Antoine Chambert-Loir, Mesures et équidistribution sur les espaces de Berkovich, J. Reine Angew. Math. 595 (2006), 215-235. 2.8

[CL11] - Heights and measures on analytic spaces. A survey of recent results, and some remarks, Motivic integration and its interactions with model theory and non-Archimedean geometry. Volume II, London Math. Soc. Lecture Note Ser., vol. 384, Cambridge Univ. Press, Cambridge, 2011, pp. $1-50.2 .8$

[Col85] Robert F. Coleman, Effective Chabauty, Duke Math. J. 52 (1985), no. 3, 765-770. 1.1

[Col87] _ Ramified torsion points on curves, Duke Math. J. 54 (1987), no. 2, 615-640. 1, 1]

[Col89] _ Reciprocity laws on curves, Compositio Math. 72 (1989), no. 2, 205-235. 1.1, 2.20, 2.6. 2.24, 2.6

[Col92] Pierre Colmez, Périodes p-adiques des variétés abéliennes, Math. Ann. 292 (1992), no. 4, 629644. 3.3

[CTT16] Adina Cohen, Michael Temkin, and Dmitri Trushin, Morphisms of Berkovich curves and the different function, Adv. Math. 303 (2016), 800-858. MR 3552539 1

[DM69] Pierre Deligne and David Mumford, The irreducibility of the space of curves of given genus, Inst. Hautes Études Sci. Publ. Math. (1969), no. 36, 75-109. 5.2

[dS06] Ehud de Shalit, Coleman integration versus Schneider integration on semistable curves, Doc. Math. (2006), no. Extra Vol., 325-334 (electronic). 3.2

[Hin88] Marc Hindry, Autour d'une conjecture de Serge Lang, Invent. Math. 94 (1988), no. 3, 575-603. 1]

[Ho14] Wei Ho, How many rational points does a random curve have?, Bull. Amer. Math. Soc. (N.S.) 51 (2014), no. 1, 27-52. 1.5

[KZB13] Eric Katz and David Zureick-Brown, The Chabauty-Coleman bound at a prime of bad reduction and Clifford bounds for geometric rank functions, Compos. Math. 149 (2013), no. 11, 1818-1838. 1.1

[Liu02] Qing Liu, Algebraic geometry and arithmetic curves, Oxford Graduate Texts in Mathematics, vol. 6, Oxford University Press, Oxford, 2002, Translated from the French by Reinie Erné, Oxford Science Publications. 2.4.3

[LT02] Dino Lorenzini and Thomas J. Tucker, Thue equations and the method of Chabauty-Coleman, Invent. Math. 148 (2002), no. 1, 47-77. 1.1

[MP12] William McCallum and Bjorn Poonen, The method of Chabauty and Coleman, Explicit methods in number theory, Panor. Synthèses, vol. 36, Soc. Math. France, Paris, 2012, pp. 99-117. 1.1

[MZ08] Grigory Mikhalkin and Ilia Zharkov, Tropical curves, their Jacobians and theta functions, Curves and abelian varieties, Contemp. Math., vol. 465, Amer. Math. Soc., Providence, RI, 2008, pp. 203230. 3.5.1, 3.27, 3.30

[Par14] Jennifer Mun Young Park, Effective Chabauty for symmetric powers of curves, Ph.D. thesis, Massachusetts Institute of Technology, 2014.1.1

[PS14] Bjorn Poonen and Michael Stoll, Most odd degree hyperelliptic curves have only one rational point, Ann. of Math. (2) 180 (2014), no. 3, 1137-1166.1.5

[PZ08] Jonathan Pila and Umberto Zannier, Rational points in periodic analytic sets and the ManinMumford conjecture, Atti Accad. Naz. Lincei Cl. Sci. Fis. Mat. Natur. Rend. Lincei (9) Mat. Appl. 19 (2008), no. 2, 149-162. 1

[Ray83] Michel Raynaud, Courbes sur une variété abélienne et points de torsion, Invent. Math. 71 (1983), no. 1, 207-233. 1, 1

[Sik09] Samir Siksek, Chabauty for symmetric powers of curves, Algebra Number Theory 3 (2009), no. 2, 209-236. 1.1 
[Sto06] Michael Stoll, Independence of rational points on twists of a given curve, Compos. Math. 142 (2006), no. 5, 1201-1214. 1.1, 4.5, 4.1

[Sto13]__ Uniform bounds for the number of rational points on hyperelliptic curves of small MordellWeil rank, preprint arXiv:1307.1773. To appear in the Journal of the European Math Society (2013). 1, 1.1, 4, 6, 1.1, 3.5.2, 3.30, 4.2, 4.3, 5.1, 5.1, 5.4, 5.2

[SW13] Arul Shankar and Xiaoheng Wang, Average size of the 2-Selmer group of Jacobians of monic even hyperelliptic curves, preprint arXiv:1307.3531 (2013). 1.5

[SZ95] Alice Silverberg and Yuri G. Zarhin, Semistable reduction and torsion subgroups of abelian varieties, Ann. Inst. Fourier (Grenoble) 45 (1995), no. 2, 403-420. 5.2

[Tem14] Michael Temkin, Metrization of differential pluriforms on Berkovich analytic spaces, preprint arXiv:1410.3079 (2014). 2.16

[Tho15] Jack A. Thorne, $E_{6}$ and the arithmetic of a family of non-hyperelliptic curves of genus 3, Forum Math. Pi 3 (2015), e1, 41. 1.5

[Thu05] A. Thuillier, Théorie du potentiel sur les courbes en géométrie analytique non archimédienne. Applications à la théorie d'Arakelov, Ph.D. thesis, University of Rennes, 2005, Preprint available at http://tel .archives-ouvertes .fr/docs/00/04/87/50/PDF/tel-00010990.pdf 2.3

[Ul198] Emmanuel Ullmo, Positivité et discrétion des points algébriques des courbes, Ann. of Math. (2) 147 (1998), no. 1, 167-179. 1

[Vol03] Vadim Vologodsky, Hodge structure on the fundamental group and its application to p-adic integration, Mosc. Math. J. 3 (2003), no. 1, 205-247, 260. 3.3

[Zar96] Yuri G. Zarhin, p-adic abelian integrals and commutative Lie groups, J. Math. Sci. 81 (1996), no. 3, 2744-2750, Algebraic geometry, 4. 3.3, 3.3, 3.4.2, 3.19, 3.5

[Zha93] Shouwu Zhang, Admissible pairing on a curve, Invent. Math. 112 (1993), no. 1, 171-193. 2.8

Department of Mathematics, The Ohio State University, Columbus, Ohio, USA

E-mail address: katz.60@osu.edu

Department of Mathematics, Georgia Tech, Atlanta, Georgia, Usa

E-mail address: rabinoff@math.gatech.edu

Department of Mathematics and Computer Science, Emory University, Atlanta, Georgia, USA

E-mail address: dzb@mathcs.emory.edu 